\title{
Selectivity Enhancement of Silica-Supported Sulfonic Acid Catalysts in Water by Coating of Ionic Liquid
}

\author{
Yanlong Gu, Ayman Karam, François Jérôme* and Joël Barrault \\ Laboratoire de Catalyse en Chimie Organique, UMR 6503, CNRS-Université de Poitiers, ESIP, 40 Avenue \\ du Recteur, Poitiers Cedex, 86022, France \\ Email Address: francois.jerome@univ-poitiers.fr
}

\section{Supporting Information}

\section{General remarks}

Silica gel (60 ACC 40-63 $\mu \mathrm{m}$, surface area: $550 \mathrm{~m}^{2} / \mathrm{g}$ ) was purchased from CARLO ERBA REACTIFS-SDS. 3-Mercaptopropyltrimethoxy silane, formalin (37\%, w/w), 1-methylimidazole, bromooctane, bis(trifluoromethane)sulfonimide lithium salt, hydrogen peroxide aqueous solution (30\%), 1-totyl-1-ethanol, 1-phenyl-1-propanol, 4-methylstyrene, 4-chlorostyrene, $\alpha$-methylstyrene, 2-phenyl-2-propanol, 2-(4-chlorophenyl)propanol, 1-phenyl-1-cyclohexene, 1,2-dodecene oxide, 3-phenyl-1-propionaldehyde and $\beta$-methylstyrene were purchased from Aldrich Chemical Company. Styrene was purchased from TCI. 1,2-Epoxy-9-decene oxide, chloroacetaldehyde aqueous solution $\quad(45 \% \quad \mathrm{w} / \mathrm{w}), \quad( \pm)$-epichlorohydrin, 1-phenyl-1-cyclohexanol, 4-chloro- $\alpha$-methylstyrene and 4-tert-butylstyrene were purchased from Alfa Aesar Chemical Company. ${ }^{1} \mathrm{H}$ and ${ }^{13} \mathrm{C}$ NMR spectra were recorded on a Bruker Avance 300 DPX 300. Chemical shifts are expressed in ppm relative to $\mathrm{Me}_{4} \mathrm{Si}$ in $\mathrm{CDCl}_{3}$. IR spectra were recorded on a FT-IR Perkin Elmer (spectrum one) using ATR technology. Elemental analysis was measured on a NA 2100 Instrument. The reaction progress was monitored on a Varian 3300 GPC equipped with a BPX5 column $(12 \mathrm{~m} \times 0.22 \mathrm{~mm}$; phase thickness: $0.25 \mu \mathrm{m})$ supplied by SGE, a Flame Detector Ionization and an injector on column. Mass was recorded over a GC/MS HP5972. Ionic liquid [ $\left.\mathrm{C}_{8} \mathrm{MIm}\right] \mathrm{NTf}_{2}$ was synthesized using the methods described in previously published material. ${ }^{1}$

Synthesis of covalently anchored sulfonic acid onto silica gel ${ }^{2}$ 


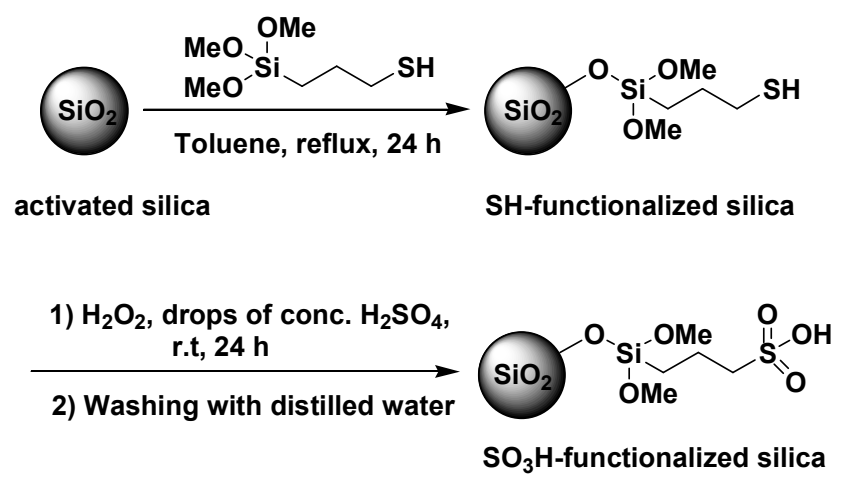

Silica was activated by an over-night heating at $150{ }^{\circ} \mathrm{C}$. In order to remove trace amount of water, toluene was treated on typical water-carry reflux equipment under refluxed condition before use. Activated silica (2 g) and 3-mercaptopropyltrimethoxy silane (0.2-0.4 g) were added into a $200 \mathrm{ml}$ of round-bottom flask containing $80-150 \mathrm{ml}$ toluene. After $24 \mathrm{~h}$ of reflux, the 3-mercaptopropyl silica was filtered off, washed with hot toluene and dried at room temperature for 2 days. The obtained 3-mercaptopropyl silica $(2 \mathrm{~g})$ was then mixed with an aqueous solution of hydrogen peroxide $(30 \%, 120 \mathrm{ml})$. The mixture was stirred at room temperature for $12 \mathrm{~h}$ after addition of three drops of conc. $\mathrm{H}_{2} \mathrm{SO}_{4}$. The solid was then filtered off and washed with distilled water till washings were neutral. Determination of ion exchange capacity was measured by our previous reported method. ${ }^{3}$

\section{Procedure for Silica-SO ${ }_{3} \mathrm{H}-\mathrm{IL}$ Catalyst Preparation. ${ }^{4}$}

The silica-supported sulfonic acid catalyst $(50 \mathrm{mg}$ ) was mixed in a $10 \mathrm{~mL}$ flask with an acetonitrile solution of ionic liquid ( $25 \mathrm{mg}$ ionic liquid was contained in this solution) at room temperature. The solution was stirred for $10 \mathrm{~min}$, and acetonitrile was then evaporated to dryness in an evaporator under reduced pressure. Finally, the obtained solid was dried in vacuo for $20 \mathrm{~min}$ to give a free-flowing white powder (50 wt $\%$ of ionic liquid was coated onto the silica gel catalyst).

\section{A typical procedure for Prins Reaction}

All reactions were conducted in a $10 \mathrm{~mL}$ flask with equipped with magnetic stirring. In a typical reaction, Silica-SO $\mathrm{SO}_{3} \mathrm{H}-\mathrm{IL}(120 \mathrm{mg})$ was mixed with $\alpha$-methylstyrene $(90 \mathrm{mg}, 0.77 \mathrm{mmol})$ and formalin $(37 \% \mathrm{w} / \mathrm{w}, 0.25 \mathrm{~mL}, 3.1 \mathrm{mmol})$ under air. The mixture was stirred for 4 hours at $90{ }^{\circ} \mathrm{C}$. After reaction, the mixture was extracted with a mixture of ethyl acetate and heptane $(1: 6 \mathrm{v} / \mathrm{v}, 6 \mathrm{~mL}$ $\times 2$ ). After concentration of organic phase, the crude compounds were purified by silica column 
chromatography to give 4-methyl-4-phenyl)-1,3-dioxane (129 mg, 94\%). The residue solid was treated under reduced pressure to removal all the volatile components, and then reused in the next run.

Table 1. Reuse of catalyst $\mathrm{SiO}_{2}-\mathrm{SO}_{3} \mathrm{H}-\left[\mathrm{C}_{8} \mathrm{MIm}\right] \mathrm{NTf} 2$ for Prins cyclization of $\alpha$-methylstyrene

\begin{tabular}{ccccc}
\hline Run & 1 & 2 & 3 & 4 \\
\hline Yield (\%) & 94 & 93 & 95 & 94 \\
\hline
\end{tabular}

4-Methyl-4-phenyl-1,3-dioxane ${ }^{5}$<smiles>CC1(c2ccccc2)CCOCO1</smiles>

(colorless liquid, heptane/ethyl acetate $=5 / 1,94 \%$ yield ${ }^{1} \mathrm{H}$ NMR $\delta 1.40(\mathrm{~s}, 3 \mathrm{H}), 2.04-2.27(\mathrm{~m}, 2 \mathrm{H})$, $3.63\left(\mathrm{td}, \mathrm{J}_{a}=11.2 \mathrm{~Hz}, \mathrm{~J}_{b}=2.8 \mathrm{~Hz}, 1 \mathrm{H}\right), 3.85-3.92(\mathrm{~m}, 1 \mathrm{H}), 4.67(\mathrm{~d}, \mathrm{~J}=6.6 \mathrm{~Hz}, 1 \mathrm{H}), 4.85(\mathrm{~d}, \mathrm{~J}=6.5$ $\mathrm{Hz}, 1 \mathrm{H}), 7.18-7.25(\mathrm{~m}, 1 \mathrm{H}) ; 7.29-7.32(\mathrm{~m}, 4 \mathrm{H}){ }^{13} \mathrm{C}$ NMR $\delta 31.1,33.9,62.6,74.7,88.2,124.8$, 126.0, 127.7, 143.0.

\section{4-Phenyl-1, 3-dioxane ${ }^{6}$}<smiles>c1ccc(C2CCOCO2)cc1</smiles>

$(\text { colorless liquid, heptane/ethyl acetate }=5 / 1,95 \% \text { yield })^{1} \mathrm{H}$ NMR $\delta 1.63\left(\mathrm{dt}, \mathrm{J}_{a}=13.5 \mathrm{~Hz}, \mathrm{~J}_{b}=1.32\right.$ $\mathrm{Hz}, 1 \mathrm{H}$ ), 2.00 (ddd, $\left.\mathrm{J}_{a}=12.8 \mathrm{~Hz}, \mathrm{~J}_{b}=4.8 \mathrm{~Hz}, \mathrm{~J}_{c}=1.3 \mathrm{~Hz}, 1 \mathrm{H}\right), 3.79\left(\mathrm{td}, \mathrm{J}_{a}=12.0 \mathrm{~Hz}, \mathrm{~J}_{b}=2.5 \mathrm{~Hz}\right.$, 1H), $4.12\left(\mathrm{dd}, \mathrm{J}_{a}=11.4 \mathrm{~Hz}, \mathrm{~J}_{b}=4.8 \mathrm{~Hz}, 1 \mathrm{H}\right), 4.57\left(\mathrm{dd}, \mathrm{J}_{a}=11.3 \mathrm{~Hz}, \mathrm{~J}_{b}=2.5 \mathrm{~Hz}, 1 \mathrm{H}\right), 4.81(\mathrm{~d}, \mathrm{~J}=$ $6.4 \mathrm{~Hz}, 1 \mathrm{H}), 5.14(\mathrm{~d}, \mathrm{~J}=6.3 \mathrm{~Hz}, 1 \mathrm{H}), 7.20-7.30(\mathrm{~m}, 1 \mathrm{H}) ;{ }^{13} \mathrm{C}$ NMR $\delta 34.4,67.3,79.2,94.6,126.2$, $128.2,128.9,141.9$.

4-(4-Methylphenyl)-1, 3-dioxane ${ }^{7}$<smiles>Cc1ccc(C2CCOCO2)cc1</smiles>

(colorless liquid, heptane/ethyl acetate $=5 / 1,96 \%$ yield) ${ }^{1} \mathrm{H}$ NMR $\delta 1.68\left(\mathrm{dt}, \mathrm{J}_{a}=13.0 \mathrm{~Hz}, \mathrm{~J}_{b}=\right.$ 1.4Hz, 1H), $2.01\left(\mathrm{dd}, \mathrm{J}_{a}=12.2 \mathrm{~Hz}, \mathrm{~J}_{b}=4.9 \mathrm{~Hz}, 1 \mathrm{H}\right), 2.26(\mathrm{~s}, 3 \mathrm{H}), 3.77\left(\mathrm{td}, \mathrm{J}_{a}=11.7 \mathrm{~Hz}, \mathrm{~J}_{b}=2.4 \mathrm{~Hz}\right.$, $1 \mathrm{H}), 4.10\left(\mathrm{dd}, \mathrm{J}_{a}=11.4 \mathrm{~Hz}, \mathrm{~J}_{b}=4.8 \mathrm{~Hz}, 1 \mathrm{H}\right), 4.52\left(\mathrm{dd}, \mathrm{J}_{a}=11.2 \mathrm{~Hz}, \mathrm{~J}_{b}=2.3 \mathrm{~Hz}, 1 \mathrm{H}\right), 4.80(\mathrm{~d}, \mathrm{~J}=$ 
6.3, 1H), $5.12(\mathrm{~d}, \mathrm{~J}=6.3 \mathrm{~Hz}, 1 \mathrm{H}), 7.13\left(\mathrm{dd}, \mathrm{J}_{a}=28.8 \mathrm{~Hz}, \mathrm{~J}_{b}=8.1 \mathrm{~Hz}, 4 \mathrm{H}\right) ;{ }^{13} \mathrm{C} \mathrm{NMR} \delta 20.1,32.9$, $65.9,77.6,93.1,124.7,128.1,136.5,137.5$.

\section{4-(4-Chlorophenyl)-1,3-dioxane ${ }^{8}$}<smiles>Clc1ccc(C2CCOCO2)cc1</smiles>

(colorless liquid, heptane/ethyl acetate $=5 / 1,92 \%$ yield) ${ }^{1} \mathrm{H}$ NMR $\delta 1.69\left(\mathrm{dt}, \mathrm{J}_{a}=13.5 \mathrm{~Hz}, \mathrm{~J}_{b}=0.9\right.$ $\mathrm{Hz}, 1 \mathrm{H}), 2.02\left(\mathrm{ddd}, \mathrm{J}_{a}=12.6 \mathrm{~Hz}, \mathrm{~J}_{b}=4.9 \mathrm{~Hz}, \mathrm{~J}_{c}=1.7 \mathrm{~Hz}, 1 \mathrm{H}\right), 3.85\left(\mathrm{td}, \mathrm{J}_{a}=11.9 \mathrm{~Hz}, \mathrm{~J}_{b}=2.5 \mathrm{~Hz}\right.$, 1H), $4.19\left(\mathrm{dd}, \mathrm{J}_{a}=11.5 \mathrm{~Hz}, \mathrm{~J}_{b}=4.8 \mathrm{~Hz}, 1 \mathrm{H}\right), 4.62\left(\mathrm{dd}, \mathrm{J}_{a}=11.3 \mathrm{~Hz}, \mathrm{~J}_{b}=2.5 \mathrm{~Hz}, 1 \mathrm{H}\right), 4.87(\mathrm{~d}, \mathrm{~J}=$ $6.4 \mathrm{~Hz}, 1 \mathrm{H}), 5.20(\mathrm{~d}, \mathrm{~J}=6.4 \mathrm{~Hz}, 1 \mathrm{H}), 7.25-7.35(\mathrm{~m}, 4 \mathrm{H}) ;{ }^{13} \mathrm{C} \mathrm{NMR} \delta 34.3,67.2,78.3,94.5,127.5$, $129.0,133.8,140.4$.

\section{4-(Chlorophenyl)-4-methyl-1,3-dioxane ${ }^{9}$}<smiles>CC1(c2ccc(Cl)cc2)CCOCO1</smiles>

(white solid, heptane/ethyl acetate $=5 / 1,94 \%$ yield ${ }^{1} \mathrm{H}$ NMR $\delta 1.37(\mathrm{~s}, 3 \mathrm{H}), 2.00-2.18(\mathrm{~m}, 2 \mathrm{H})$, $3.59\left(\mathrm{td}, \mathrm{J}_{a}=10.2 \mathrm{~Hz}, \mathrm{~J}_{b}=3.1 \mathrm{~Hz}, 1 \mathrm{H}\right), 3.83-3.90(\mathrm{~m}, 1 \mathrm{H}), 4.62(\mathrm{~d}, \mathrm{~J}=6.6 \mathrm{~Hz}, 1 \mathrm{H}), 4.83(\mathrm{~d}, \mathrm{~J}=6.6$ $\mathrm{Hz}, 1 \mathrm{H}), 7.17-7.28(\mathrm{~m}, 4 \mathrm{H}) ;{ }^{13} \mathrm{C}$ NMR $\delta 30.6,33.8,62.4,74.3,88.0,126.2,127.8,131.8,141.8$.

\section{4-[4-(1,1-Dimethylethyl)phenyl]-1,3-dioxane ${ }^{10}$}<smiles>CC(C)(C)c1ccc(C2CCOCO2)cc1</smiles>

(colorless liquid, heptane/ethyl acetate $=6 / 1,92 \%$ yield) ${ }^{1} \mathrm{H}$ NMR $\delta 1.31(\mathrm{~s}, 9 \mathrm{H}), 1.70\left(\mathrm{dt}, \mathrm{J}_{a}=13.5\right.$ $\left.\mathrm{Hz}, \mathrm{J}_{b}=0.8 \mathrm{~Hz}, 1 \mathrm{H}\right), 2.14\left(\mathrm{ddd}, \mathrm{J}_{a}=25.3, \mathrm{~J}_{b}=11.9 \mathrm{~Hz}, \mathrm{~J}_{c}=4.8 \mathrm{~Hz}, 1 \mathrm{H}\right), 3.86\left(\mathrm{td}, \mathrm{J}_{a}=11.9 \mathrm{~Hz}, \mathrm{~J}_{b}=\right.$ $2.4 \mathrm{~Hz}, 1 \mathrm{H}), 4.20\left(\mathrm{dd}, \mathrm{J}_{a}=11.4 \mathrm{~Hz}, \mathrm{~J}_{b}=4.8 \mathrm{~Hz}, 1 \mathrm{H}\right), 4.62\left(\mathrm{dd}, \mathrm{J}_{a}=11.3 \mathrm{~Hz}, \mathrm{~J}_{b}=2.4 \mathrm{~Hz}, 1 \mathrm{H}\right), 4.88$ $(\mathrm{d}, \mathrm{J}=6.3 \mathrm{~Hz}, 1 \mathrm{H}), 5.20(\mathrm{~d}, \mathrm{~J}=6.3 \mathrm{~Hz}, 1 \mathrm{H}), 7.34\left(\mathrm{dd}, \mathrm{J}_{a}=24.6 \mathrm{~Hz}, \mathrm{~J}_{b}=8.3 \mathrm{~Hz}, 4 \mathrm{H}\right) ;{ }^{13} \mathrm{C} \mathrm{NMR} \delta$ $31.8,34.1,35.0,67.4,79.1,94.6,125.8,126.0,138.8,151.2$.

Hexahydro-8a-phenyl-4H-1,3-dioxane ${ }^{11}$ 
<smiles>c1ccc(C23CCCCC2COCO3)cc1</smiles>

$($ white solid, heptane/ethyl acetate $=5 / 1,90 \%$ yield $){ }^{1} \mathrm{H}$ NMR $\delta 1.18-1.31(\mathrm{~m}, 2 \mathrm{H}), 1.34-1.47(\mathrm{~m}$, $1 \mathrm{H}), 1.60-1.70(\mathrm{~m}, 2 \mathrm{H}), 1.75-1.80(\mathrm{~m}, 2 \mathrm{H}), 2.10\left(\mathrm{dt}, \mathrm{J}_{a}=12.1 \mathrm{~Hz}, \mathrm{~J}_{b}=2.2 \mathrm{~Hz}, 1 \mathrm{H}\right), 2.29\left(\mathrm{ddd}, \mathrm{J}_{a}=\right.$ $\left.25.4 \mathrm{~Hz}, \mathrm{~J}_{b}=17.4 \mathrm{~Hz}, \mathrm{~J}_{c}=4.7 \mathrm{~Hz}, 1 \mathrm{H}\right), 3.48(\mathrm{~d}, \mathrm{~J}=11.1 \mathrm{~Hz}, 1 \mathrm{H}), 3.77\left(\mathrm{dd}, \mathrm{J}_{a}=11.1 \mathrm{~Hz}, \mathrm{~J}_{b}=2.1 \mathrm{~Hz}\right.$, 1H), $4.77\left(\mathrm{dd}, \mathrm{J}_{a}=24.4 \mathrm{~Hz}, \mathrm{~J}_{b}=6.3 \mathrm{~Hz}, 1 \mathrm{H}\right), 7.16-7.25(\mathrm{~m}, 1 \mathrm{H}), 7.30(\mathrm{~d}, \mathrm{~J}=4.3 \mathrm{~Hz}, 4 \mathrm{H}) ;{ }^{13} \mathrm{C} \mathrm{NMR}$ $\delta 20.9,24.4,25.0,34.9,42.4,67.4,76.3,88.5,124.8,125.8,127.7,144.0$.

\section{5-Methyl-4-phenyl-1,3-dioxane ${ }^{12}$}<smiles>CC1COCOC1c1ccccc1</smiles>

(colorless liquid, heptane/ethyl acetate $=5 / 1,89 \%$ yield) ${ }^{1} \mathrm{H}$ NMR $\delta 0.47(\mathrm{~d}, \mathrm{~J}=6.8 \mathrm{~Hz}, 1.8 \mathrm{H})$, $0.80\left(\mathrm{dd}, \mathrm{J}_{a}=7.0 \mathrm{~Hz}, \mathrm{~J}_{b}=0.3 \mathrm{~Hz}, 1.2 \mathrm{H}\right), 1.68-1.76(\mathrm{~m}, 0.4 \mathrm{H}), 1.96-2.07(\mathrm{~m}, 0.6 \mathrm{H}), 3.23(\mathrm{t}, \mathrm{J}=$ 11.1 Hz, 0.6 H), 3.81-4.01 (m, 2 H), 4.70-4.77 (m, 1.4 H), 5.08-5.14 (m, 1H), 7.11-7.27 (m, 5 H); ${ }^{13} \mathrm{C}$ NMR $\delta 10.1,11.4,33.8,35.4,71.9,71.9,79.4,85.1,93.0,93.5,124.0,125.9,126.3,127.1$, $127.2,127.3,138.5,139.5$.

\section{A typical procedure for tandem dehydration/Prins Reaction}

In a typical reaction, Silica-SO ${ }_{3} \mathrm{H}-\mathrm{IL}(120 \mathrm{mg})$ was mixed with 2-phenyl-2-propanol (105 mg, 0.77 mmol) and formalin $(37 \% \mathrm{w} / \mathrm{w}, 0.25 \mathrm{~mL}, 3.1 \mathrm{mmol})$ under air. The mixture was stirred for 4.5 hours at $90{ }^{\circ} \mathrm{C}$. After reaction, the mixture was extracted with a mixture of ethyl acetate and heptane $(1: 6 \mathrm{v} / \mathrm{v}, 6 \mathrm{~mL} \times 2)$. After concentration of organic phase, the crude compounds were purified by silica column chromatography to give 4-methyl-4-phenyl)-1,3-dioxane (126 mg, 92\%).

\section{A typical procedure for cycloaddition of formaldehyde to epoxide}

In a typical reaction, Silica- $\mathrm{SO}_{3} \mathrm{H}-\mathrm{IL}(120 \mathrm{mg})$ was mixed with 1,2-dodecene oxide (198 mg, 1.1 $\mathrm{mmol})$ and formalin $(37 \% \mathrm{w} / \mathrm{w}, 0.25 \mathrm{~mL}, 3.1 \mathrm{mmol})$ under air. The mixture was stirred for 4 hours at $90{ }^{\circ} \mathrm{C}$. After reaction, the mixture was extracted with a mixture of ethyl acetate and heptane $(1: 6$ $\mathrm{v} / \mathrm{v}, 6 \mathrm{~mL} \times 2$ ). After concentration of organic phase, the crude compounds were purified by silica 
column chromatography to give 4-Decyl-1,3-dioxolane (212 mg, 90\%). When chloroacetaldehyde was used, $0.25 \mathrm{~mL}$ of the aqueous solution was charged for $1.1 \mathrm{mmol}$ of reaction. In case of 3-phenylpropionaldehyde, $1.1 \mathrm{mmol}$ of aldehyde and $0.30 \mathrm{~mL}$ of distilled water were used as solvent. For the reaction between epichlorohydrin and 3-phenylpropionaldehyde, the epoxide was used in excess amount (1.5 equiv relative to the aldehyde).

\section{4-Decyl-1,3-dioxolane ${ }^{13}$}<smiles>CCCCCCCCCCC1COCO1</smiles>

(colorless liquid, heptane/ethyl acetate $=4 / 1,90 \%$ yield) ${ }^{1} \mathrm{H}$ NMR $\delta 0.81(\mathrm{t}, \mathrm{J}=6.4 \mathrm{~Hz}, 3 \mathrm{H})$, 1.19-1.61 (m, 18H), $3.35(\mathrm{t}, \mathrm{J}=20 \mathrm{~Hz}, 1 \mathrm{H}), 3.87-3.96(\mathrm{~m}, 2 \mathrm{H}), 4.75(\mathrm{~s}, 1 \mathrm{H}), 4.95(\mathrm{~s}, 1 \mathrm{H}),{ }^{13} \mathrm{C} \mathrm{NMR}$ $\delta 13.1,21.7,24.8,28.3,28.5,28.5,28.6,30.9,32.0,68.6,75.3,93.8$.

\section{2-(Chloromethyl)-4-decyl-1,3-dioxolane ${ }^{14}$}

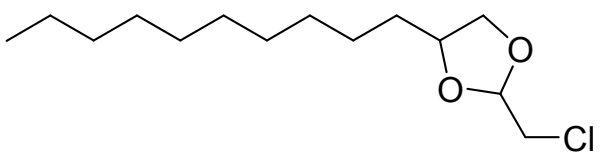

(colorless liquid, heptane/ethyl acetate $=4 / 1,92 \%$ yield) ${ }^{1} \mathrm{H}$ NMR $\delta 0.81(\mathrm{t}, \mathrm{J}=6.5 \mathrm{~Hz}, 3 \mathrm{H})$, 1.19-1.63 (m, 18H), 3.42-3.52 (m, 3H), 3.92-4.09 (m, 2H), 5.07 (t, J = 4.0 Hz, 0.8 H), 5.19 (t, J =

$4.1 \mathrm{~Hz}, 0.2 \mathrm{H}),{ }^{13} \mathrm{C}$ NMR $\delta 14.5,23.1,26.0,26.1,29.7,29.9,30.0,30.0,32.3,33.2,33.2,45.1,45.2$, $78.1,102.5,102.9$.

\section{2-(2-Phenylethyl)-4-decyl-1,3-dioxolane}

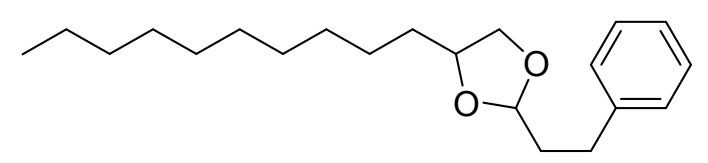

(colorless liquid, heptane/ethyl acetate $=5 / 1,93 \%$ yield) ${ }^{1} \mathrm{H}$ NMR $\delta 0.80(\mathrm{t}, \mathrm{J}=6.4 \mathrm{~Hz}, 3 \mathrm{H})$, 1.18-1.60 (m, 18H), 1.84-1.99 (m, 2H), 2.58-2.72 (s, 2H), 3.32-3.43 (m, 1H), 3.83-4.00 (m, 2H), $4.88\left(\mathrm{dt}, \mathrm{J}_{a}=21.9 \mathrm{~Hz}, \mathrm{~J}_{b}=4.7 \mathrm{~Hz}, 1 \mathrm{H}\right), 7.10-7.23(\mathrm{~m}, 5 \mathrm{H}),{ }^{13} \mathrm{C} \mathrm{NMR} \delta 13.1,21.7,24.7,28.3,28.5$, 28.6, 28.6, 29.0, 29.1, 31.0, 32.3, 32.5, 34.7, 34.8, 68.7, 69.6, 75.2, 75.9, 102.0, 102.7, 124.8, 127.3, 127.4, 140.7, 140.7; MS (EI) m/z (relative intensity) 91, 55, 69, 83, 213, 111, 240, 317[ $\left.\mathrm{M}^{+}-1\right]$, 318[M $\mathrm{M}^{+}$; IR: 2923.6, 2853.9, 1455.6, 1136.9, 1030.3, $695.5 \mathrm{~cm}^{-1}$; Anal. Calcd for $\mathrm{C}_{21} \mathrm{H}_{34} \mathrm{O}_{2}: \mathrm{C}$, 79.19; H, 10.76; O, 10.05. Found: C, 78.92, H, 10.54; O, 10.13 .

4-(Chloromethyl)-2-(2-phenylethyl)-1,3-dioxolane ${ }^{15}$ 


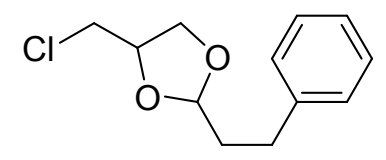

(colorless liquid, heptane/ethyl acetate $=5 / 1,89 \%$ yield) ${ }^{1} \mathrm{H}$ NMR $\delta$ 1.85-1.95 $(\mathrm{m}, 2 \mathrm{H}), 2.61-2.69$ $(\mathrm{m}, 2 \mathrm{H}), 3.32-3.57(\mathrm{~m}, 2 \mathrm{H}), 3.62-3.68\left(\mathrm{dd}, \mathrm{J}_{a}=8.6 \mathrm{~Hz}, \mathrm{~J}_{b}=6.4 \mathrm{~Hz}, 0.5 \mathrm{H}\right), 3.84-3.89(\mathrm{~m}, 1 \mathrm{H})$, 4.10-4.36 (m, 1.5H), $4.92\left(\mathrm{dt}, \mathrm{J}_{a}=33.7 \mathrm{~Hz}, \mathrm{~J}_{b}=4.7 \mathrm{~Hz}, 1 \mathrm{H}\right), 7.10-7.22(\mathrm{~m}, 5 \mathrm{H}),{ }^{13} \mathrm{C}$ NMR $\delta 30.3$, $30.4,35.9,44.6,44.9,68.7,69.2,75.5,75.8,104.8,105.3,126.4,128.8,128.9,141.7,141.8$.

\section{4-(7-Octenyl)-1,3-dioxolane}

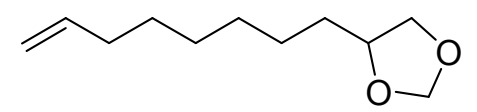

$($ colorless liquid, heptane/ethyl acetate $=5 / 1,94 \%$ yield $){ }^{1} \mathrm{H}$ NMR $\delta 1.27-1.68(\mathrm{~m}, 10 \mathrm{H}), 2.01-2.08$ (m, 2H), 3.38-3.45 (m, 1H), 3.93-4.03 (m, 2H), 4.87-5.01 (m, 4H), 5.74-5.87 (m, 1H), ${ }^{13} \mathrm{C}$ NMR $\delta$ 26.2, 29.2, 29.3, 29.8, 33.4, 34.1, 70.0, 76.7, 95.2, 114.6, 139.4; MS (EI) m/z (relative intensity) 55, 81, 67, 61, 95, 123, 185 [M+ + 1]; IR:2928, 2856, 1464, 1259, 1089, 1012, 910, $794 \mathrm{~cm}^{-1}$; Anal. Calcd for $\mathrm{C}_{11} \mathrm{H}_{20} \mathrm{O}_{2}$ : C, 71.70; H, 10.94; O, 17.36. Found: C, 71.47, H, 10.86; O, 17.14 .

\section{2-(Chloromethyl)-4-(7-octenyl)-1,3-dioxolane}

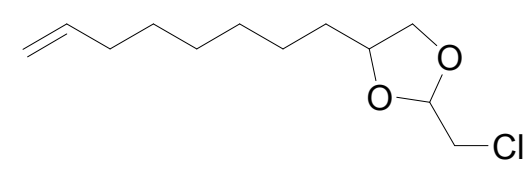

(colorless liquid, heptane/ethyl acetate $=5 / 1,95 \%$ yield $){ }^{1} \mathrm{H}$ NMR $\delta 1.17-1.65(\mathrm{~m}, 10 \mathrm{H}), 1.93-2.00$ (m, 2H), 3.36-3.54 (m, 3H), 3.90-4.16 (m, 2H), 4.83-4.85 (m, 0.4 H), 4.87-4.90 (m, 1H), 4.92-4.95 $(\mathrm{m}, 0.6 \mathrm{H}), 5.07$ (t, J = 4.0 Hz, 0.8 H), $5.16(\mathrm{t}, \mathrm{J}=4.1 \mathrm{~Hz}, 0.2 \mathrm{H}), 5.66-5.79(\mathrm{~m}, 1 \mathrm{H}),{ }^{13} \mathrm{C}$ NMR $\delta$ 24.6, 24.6, 27.8, 27.9, 28.4, 31.8, 31.8, 32.7, 43.7, 43.8, 69.1, 69.7, 76.0, 76.7, 101.1, 101.5, 113.3, 138.0; MS (EI) m/z (relative intensity) 95, 81, 55, 67, 183, 231 [M+ - 1]; IR:2928, 2857, 1430, 1141, 1044, 1009, 909, $763 \mathrm{~cm}^{-1}$; Anal. Calcd for $\mathrm{C}_{12} \mathrm{H}_{21} \mathrm{ClO}_{2}$ : C, 61.92; H, 9.09; O, 13.75. Found: $\mathrm{C}$, 61.66, H, 8.74; O, 13.83.

\section{4-(7-Octenyl)-2-(2-phenylethyl)-1,3-dioxolane}

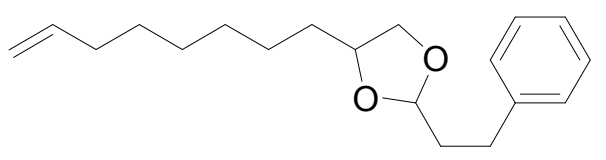

(colorless liquid, heptane/ethyl acetate $=5 / 1,93 \%$ yield $){ }^{1} \mathrm{H}$ NMR $\delta 1.19-1.57(\mathrm{~m}, 10 \mathrm{H}), 1.84-1.99$ (m, 4H), 2.63-2.70 (m, 2H), 3.32-3.45 (m, 1H), 3.82-4.07 (m, 2H), 4.84-4.95 (m, 3H), 5.66-5.80 (m, 1H), 7.06-7.22 (m, 5H), ${ }^{13} \mathrm{C}$ NMR $\delta 24.7,27.8,27.9,28.4,29.0,29.1,32.3,32.4,32.7,34.7,34.8$, 
68.7, 69.6, 75.1, 75.8, 102.0, 102.7, 113.2, 124.8, 127.3, 127.4, 138.0, 140.6, 140.7; MS (EI) m/z (relative intensity) 95, 81, 55, 67, 183, 210, $287\left[\mathrm{M}^{+}-1\right], 288\left[\mathrm{M}^{+}\right]$; IR: 2927, 2856, 1455, 1135, 1030, 994, 908, 747, $698 \mathrm{~cm}^{-1}$; Anal. Calcd for $\mathrm{C}_{19} \mathrm{H}_{28} \mathrm{O}_{2}$ : C, 79.12; H, 9.78; O, 11.09. Found: C, 78.84, H, 9.57; O, 10.85 .

\section{A typical procedure for etherification of secondary alcohohs}

In a typical reaction, Silica- $\mathrm{SO}_{3} \mathrm{H}-\mathrm{IL}(120 \mathrm{mg})$ was mixed with distilled water $(0.4 \mathrm{~mL})$ and 1-totyl-1-ethanol (136 mg, $1.0 \mathrm{mmol})$ under air. The mixture was stirred for 5 hours at $80{ }^{\circ} \mathrm{C}$. After reaction, the mixture was extracted with a mixture of ethyl acetate and heptane $(1: 6 \mathrm{v} / \mathrm{v}, 8 \mathrm{~mL} \times 2)$. After concentration of organic phase, the crude compounds were purified by silica column chromatography to give 1,1'-(Oxydiethylidene)bis[4-methylbenzene] (244 mg, 96\%).

\section{1,1'-(Oxydiethylidene)bis[4-methylbenzene] ${ }^{16}$}<smiles>Cc1ccc(C(C)OC(C)c2ccc(C)cc2)cc1</smiles>

(colorless liquid, heptane/ethyl acetate $=10 / 1,96 \%$ yield) ${ }^{1} \mathrm{H}$ NMR $\delta 1.27(\mathrm{~d}, \mathrm{~J}=6.5 \mathrm{~Hz}, 3.5 \mathrm{H})$, $1.35(\mathrm{~d}, 6.4 \mathrm{~Hz}, 2.5 \mathrm{H}), 2.22(\mathrm{~s}, 2 \mathrm{H}), 2.26(\mathrm{~s}, 4 \mathrm{H}), 4.12\left(\mathrm{dd}, \mathrm{J}_{a}=13.0 \mathrm{~Hz}, \mathrm{~J}_{b}=6.5 \mathrm{~Hz}, 1.2 \mathrm{H}\right), 4.40$ $\left(\mathrm{dd}, \mathrm{J}_{a}=12.7 \mathrm{~Hz}, \mathrm{~J}_{b}=6.4 \mathrm{~Hz}, 0.8 \mathrm{H}\right), 6.99-7.14(\mathrm{~m}, 8 \mathrm{H}) ;{ }^{13} \mathrm{C} \mathrm{NMR} \delta 20.1,20.1,21.9,23.7,72.9$, $73.2,125.1,125.2,127.9,128.1,135.6,135.9,140.1,140.3$.

\section{Bis(1-phenylpropyl) ether ${ }^{17}$}<smiles>CCC(OC(CC)c1ccccc1)c1ccccc1</smiles>

(colorless liquid, heptane/ethyl acetate $=10 / 1,91 \%$ yield) ${ }^{1} \mathrm{H}$ NMR $\delta 0.70(\mathrm{t}, \mathrm{J}=7.4 \mathrm{~Hz}, 3.3 \mathrm{H}$ ), 0.77 (t, J = 7.4 Hz, 2.7 H), 1.44-1.59 (m, 0.6 H), 1.61-1.84 (m, 1.4 H), 3.86 (t, J = 6.6 Hz, 0.6 H), $4.40(\mathrm{t}, \mathrm{J}=6.2 \mathrm{~Hz}, 0.4 \mathrm{H}), 7.07-7.26(\mathrm{~m}, 5 \mathrm{H}){ }^{13} \mathrm{C} \mathrm{NMR} \delta 10.2,10.9,30.2,31.8,80.4,81.0,127.2$, 127.4, 127.6, 127.8, 128.4, 128.7, 143.3, 143.4. 


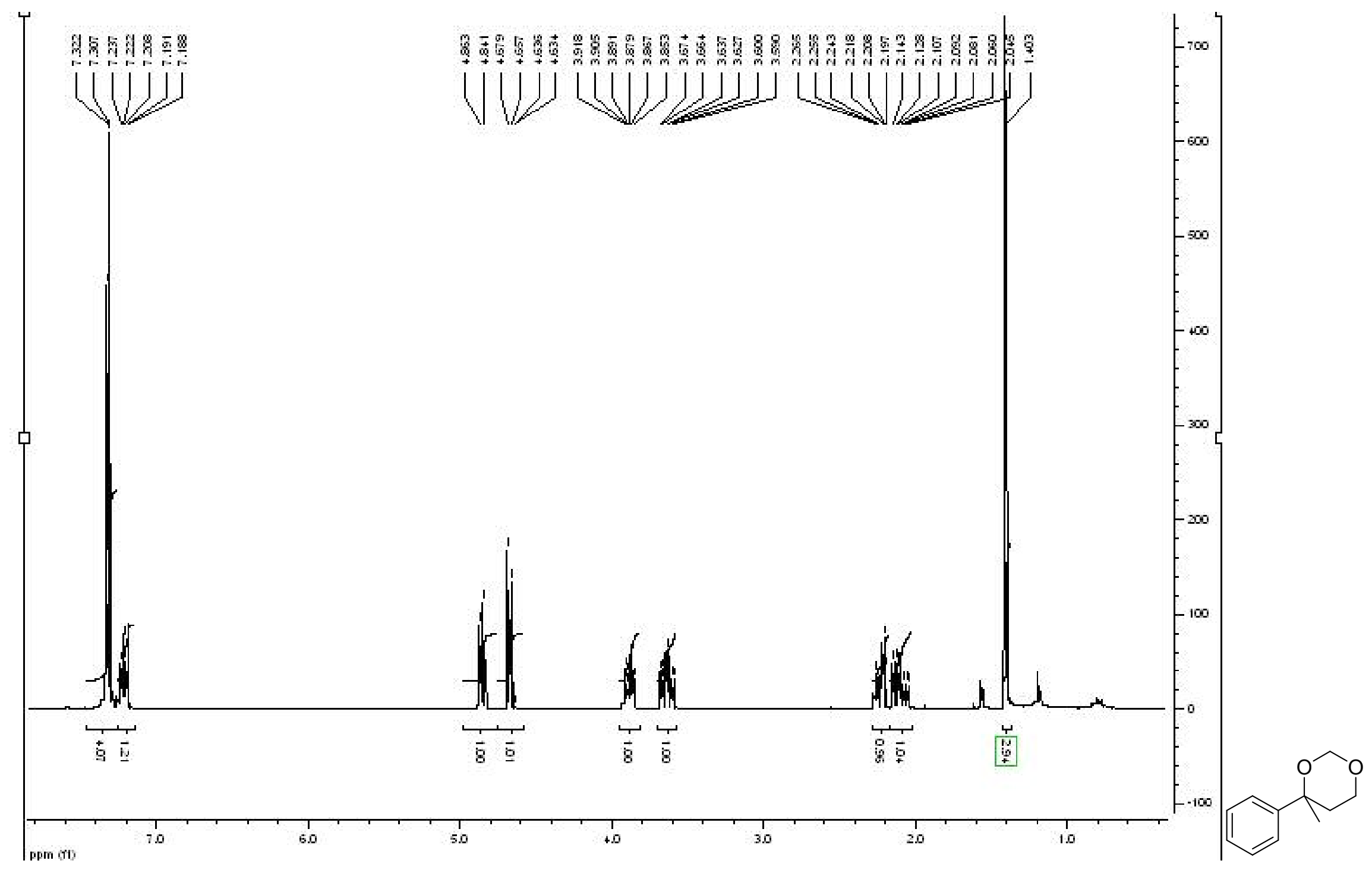




$$
\text { . }
$$




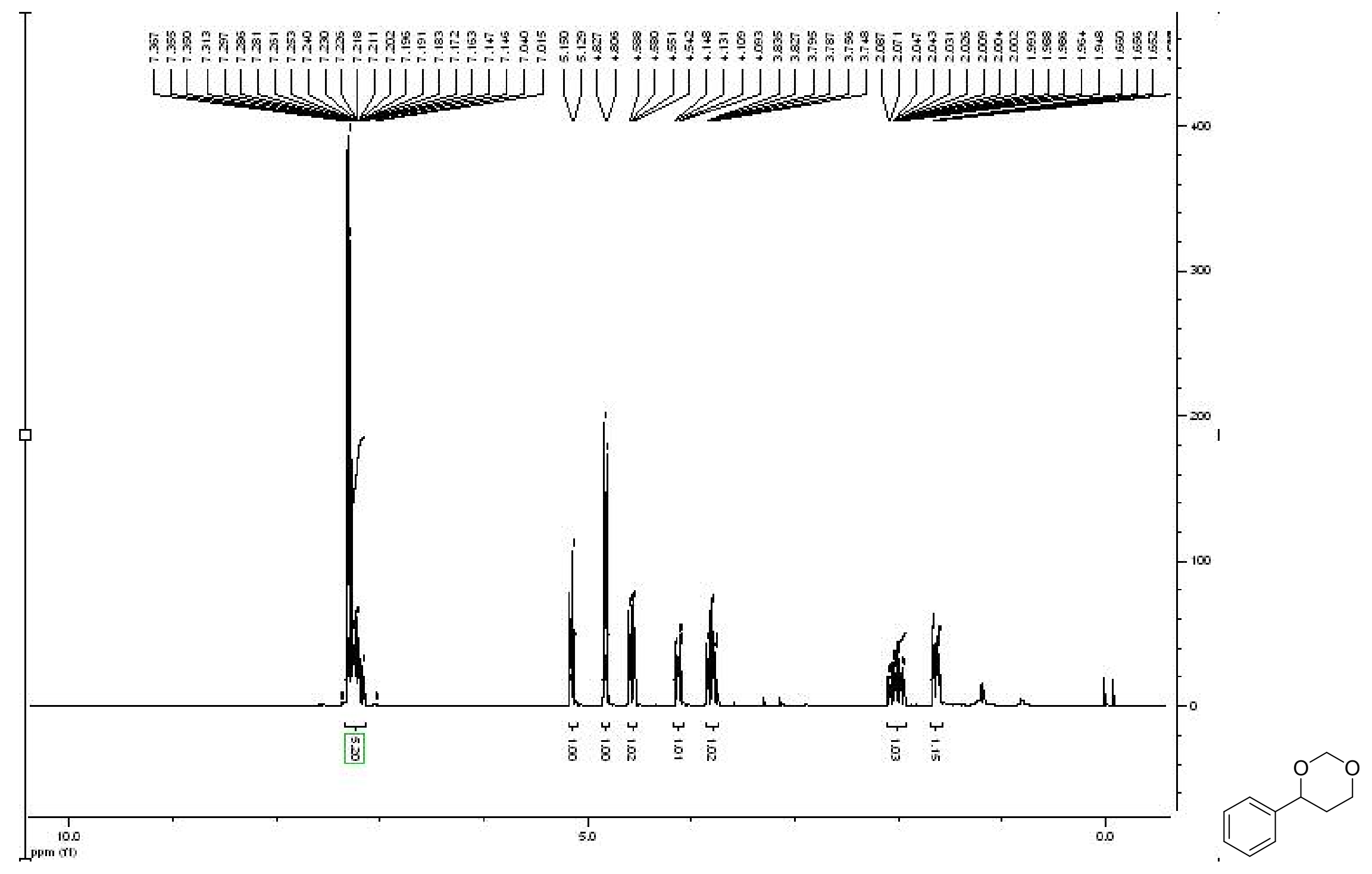



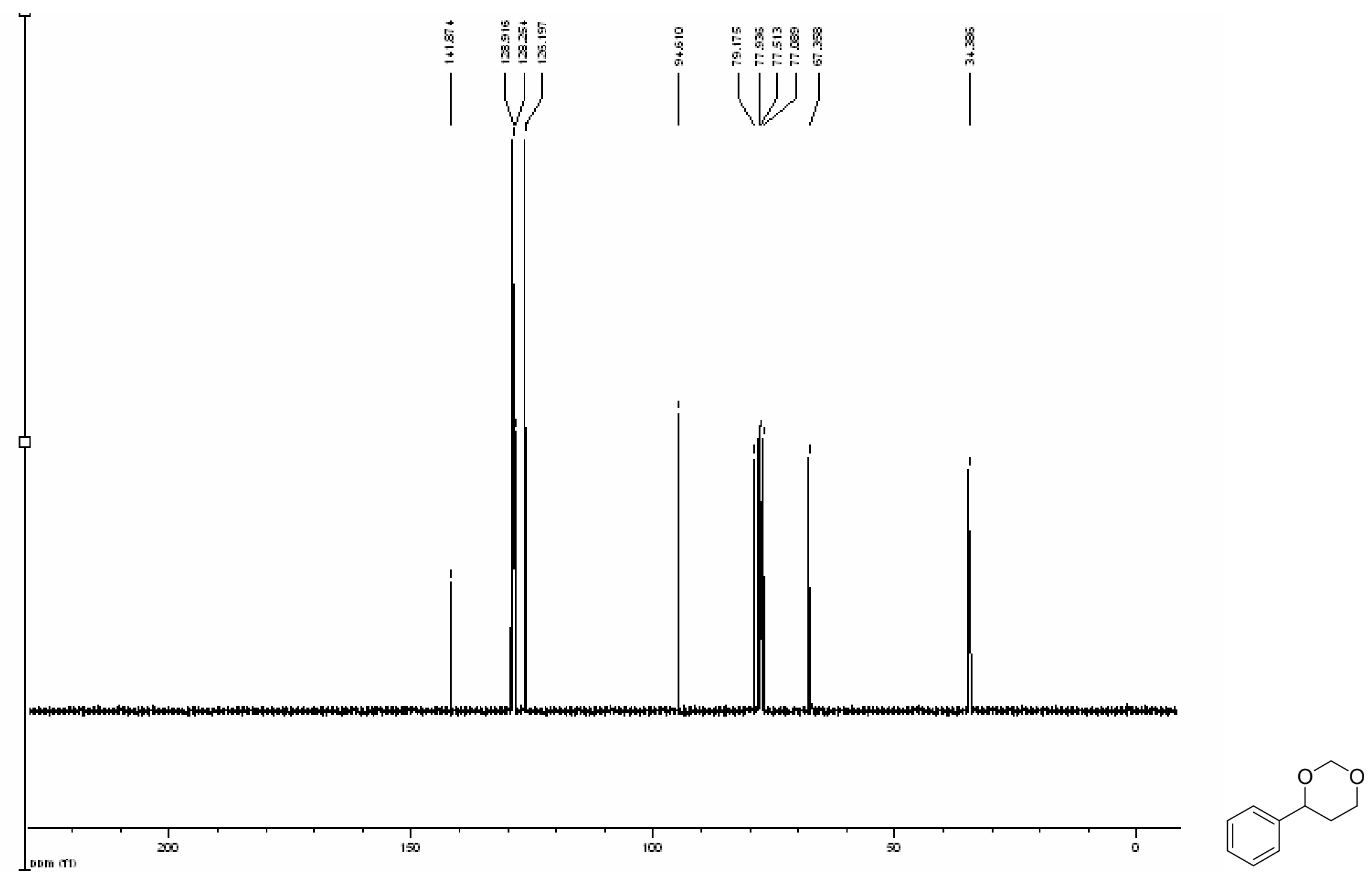


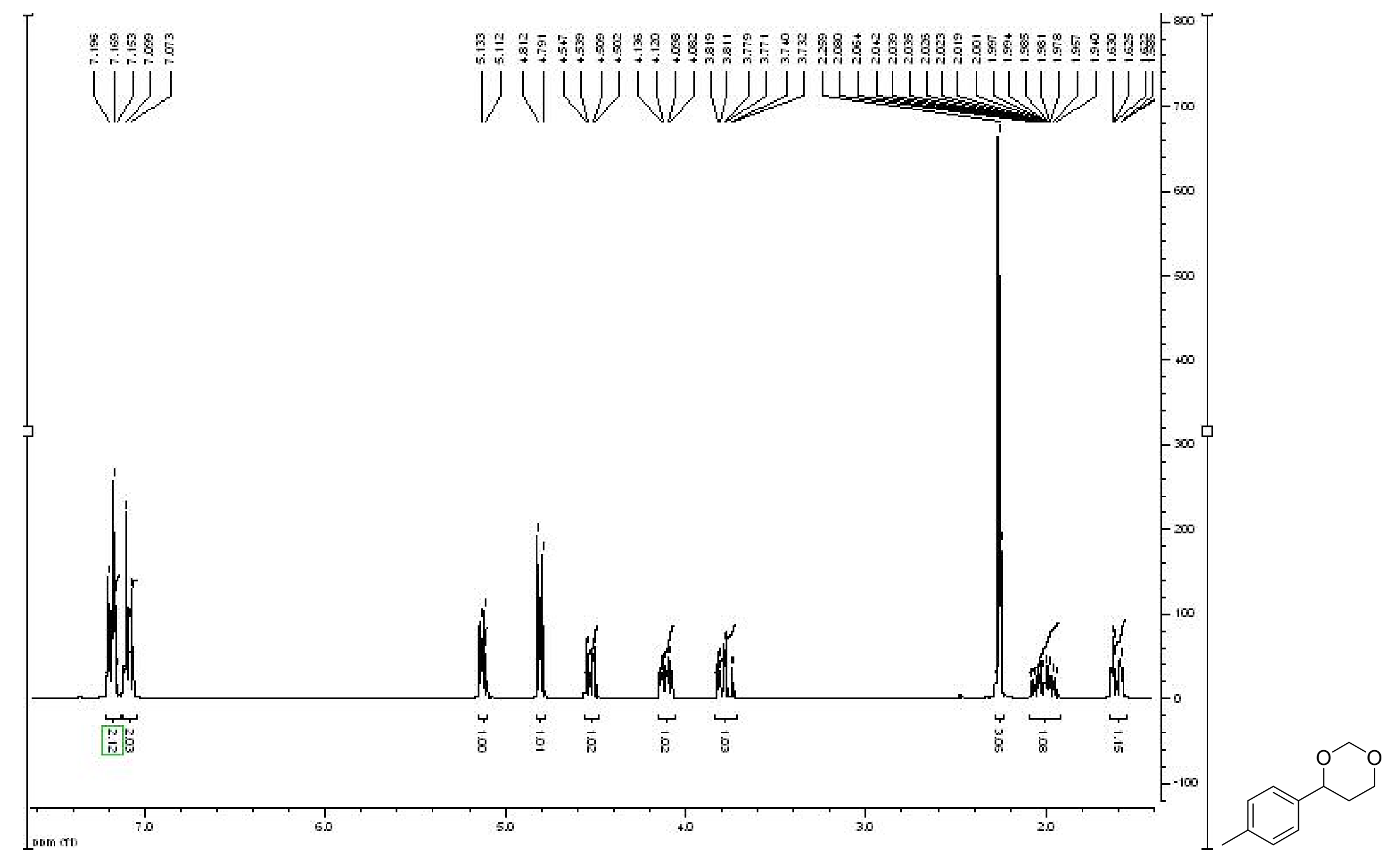



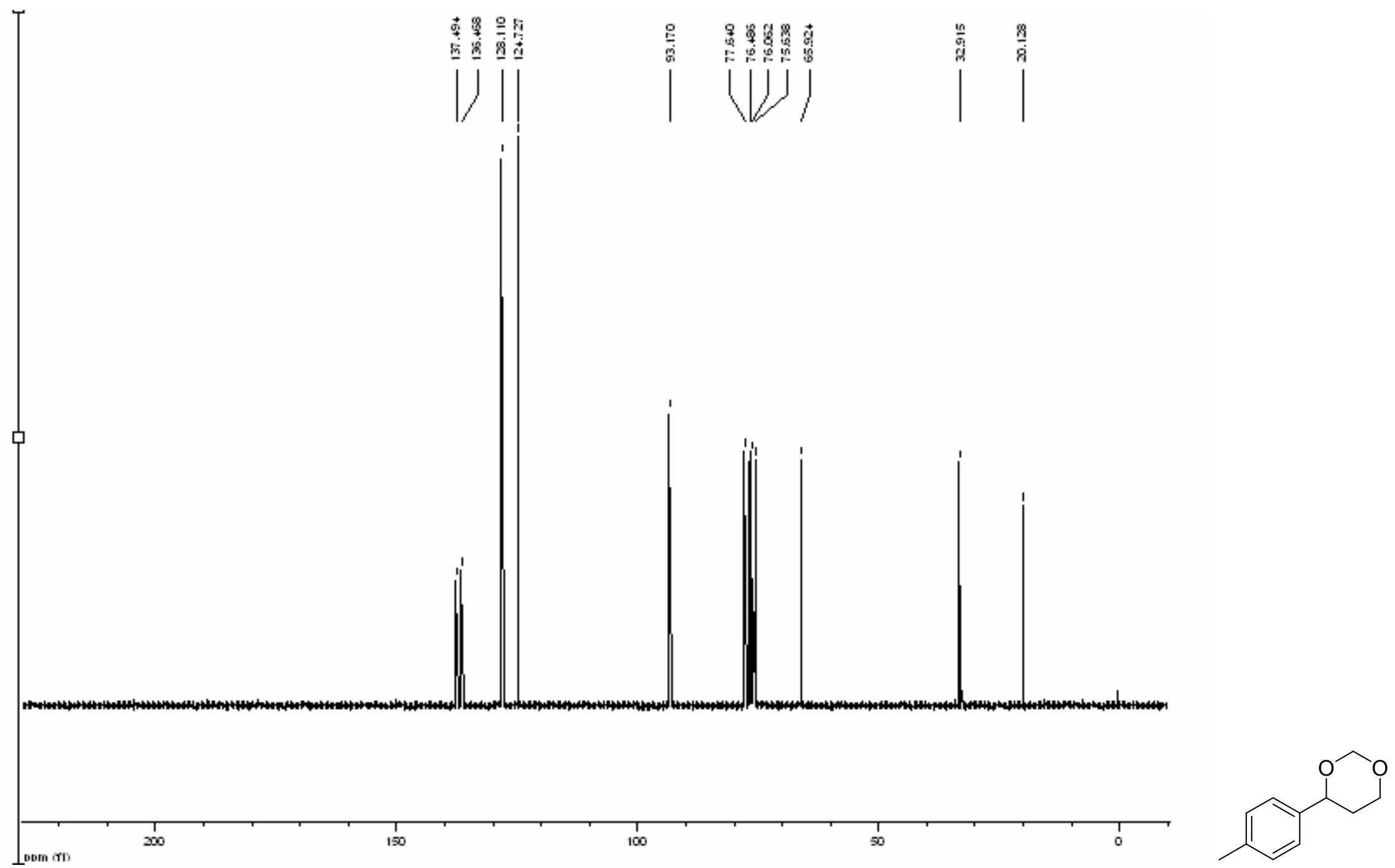


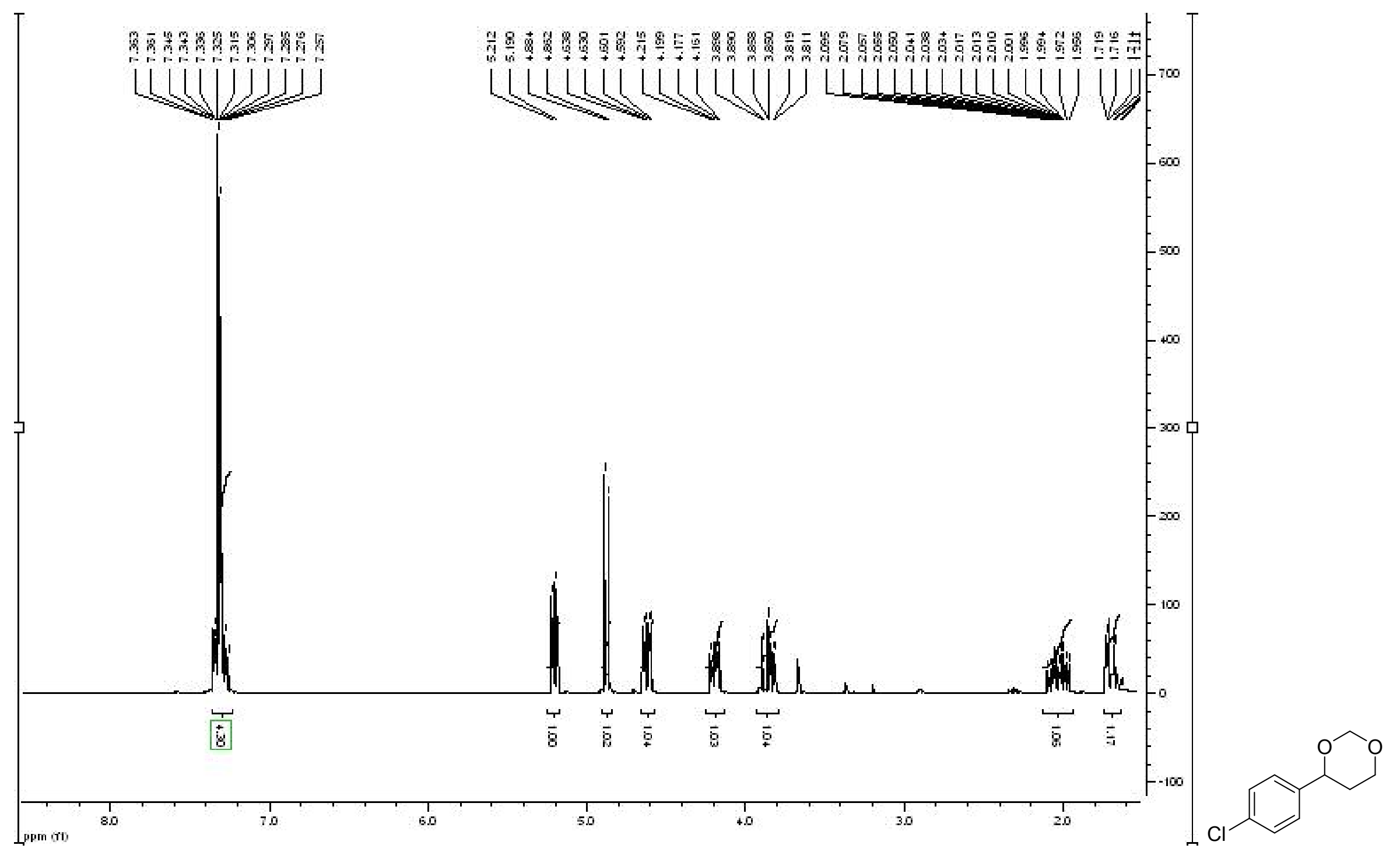




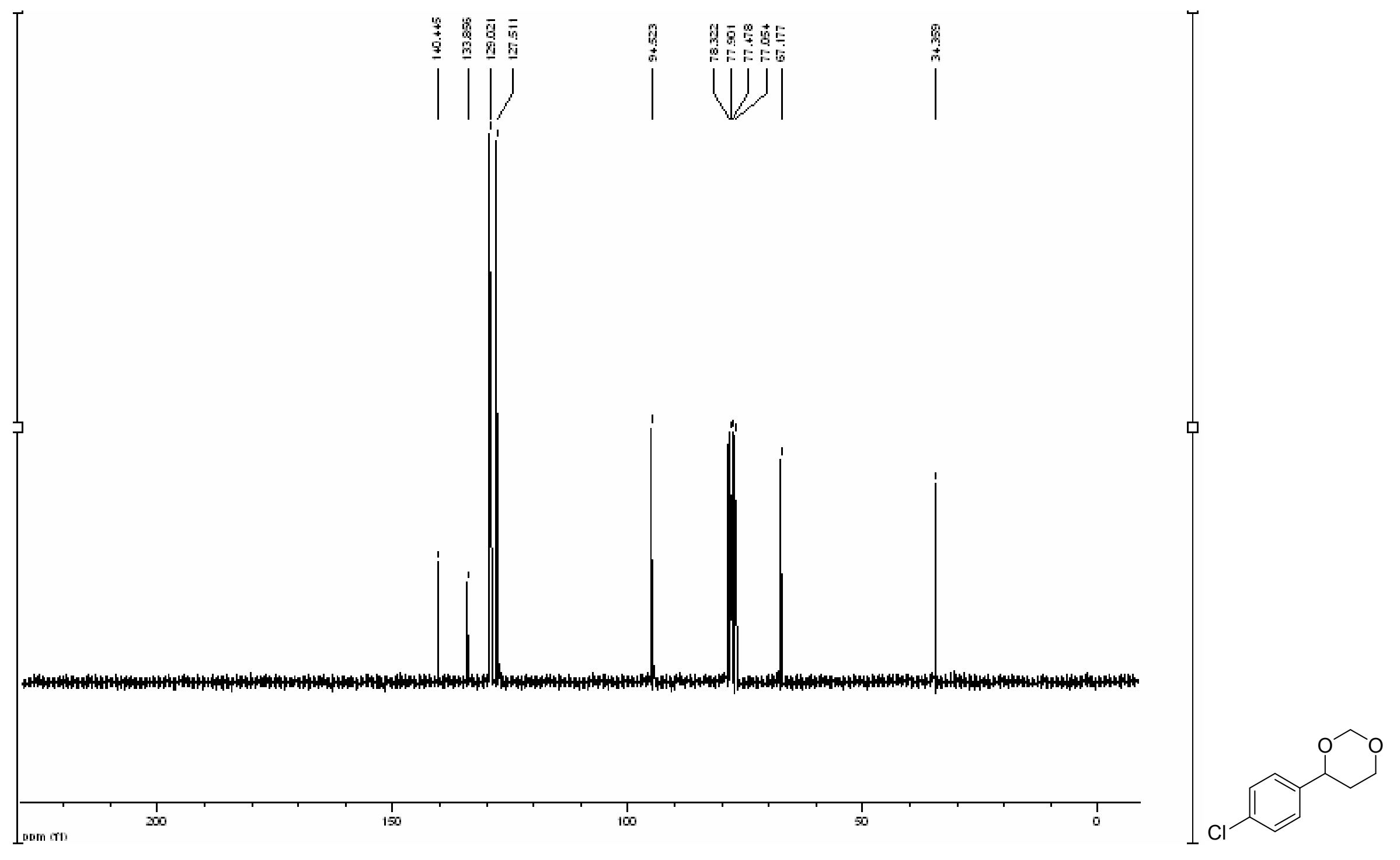




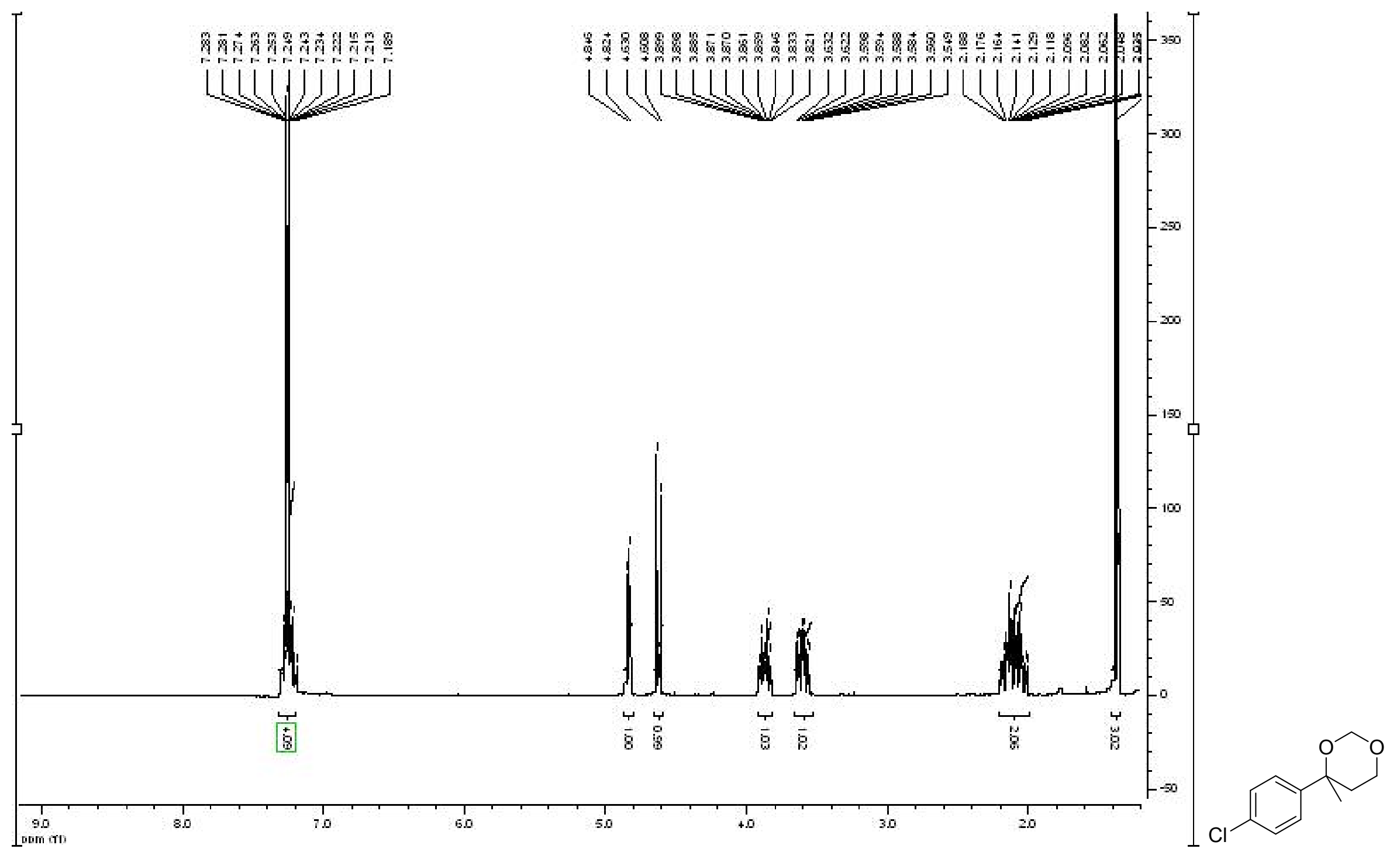




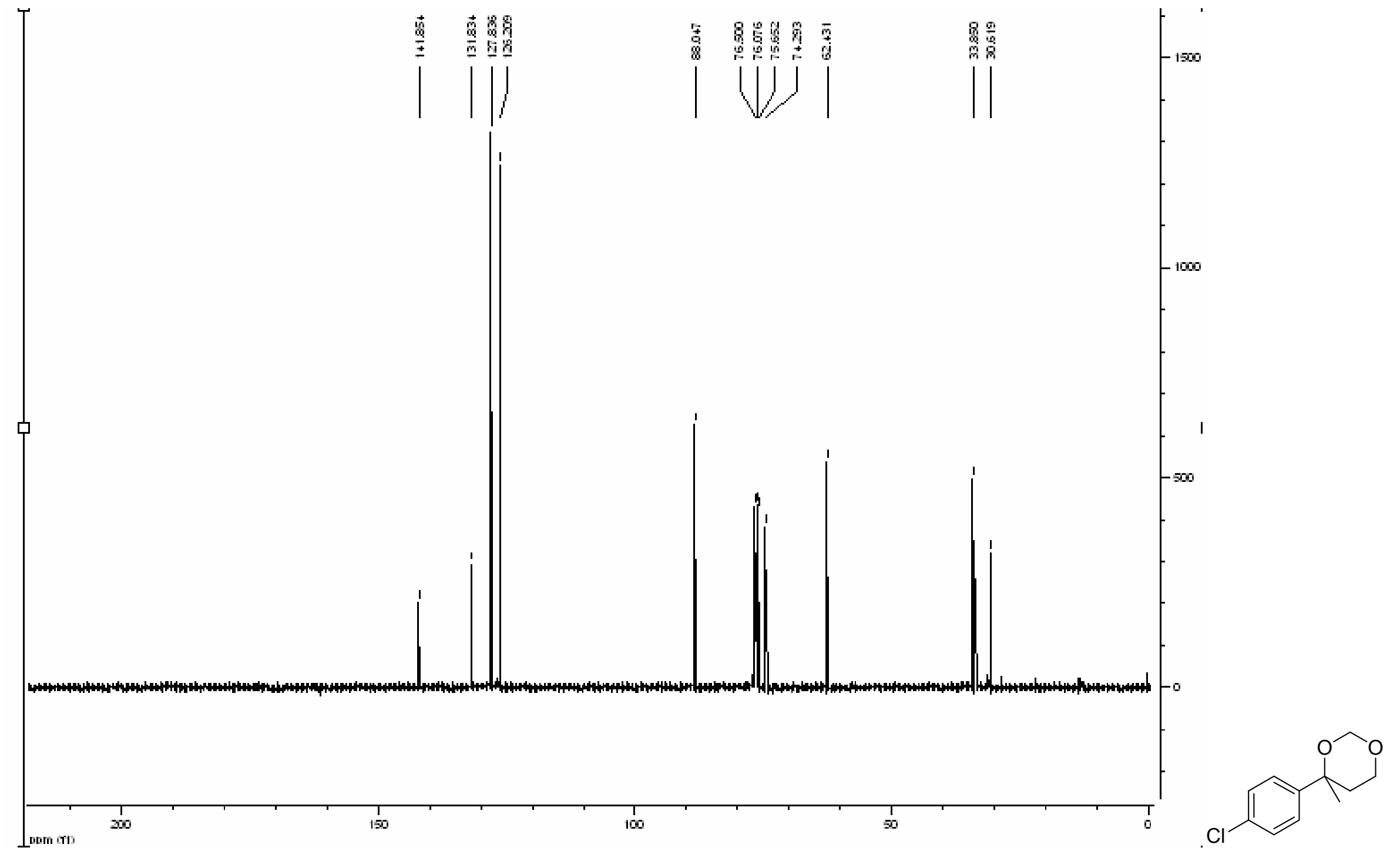




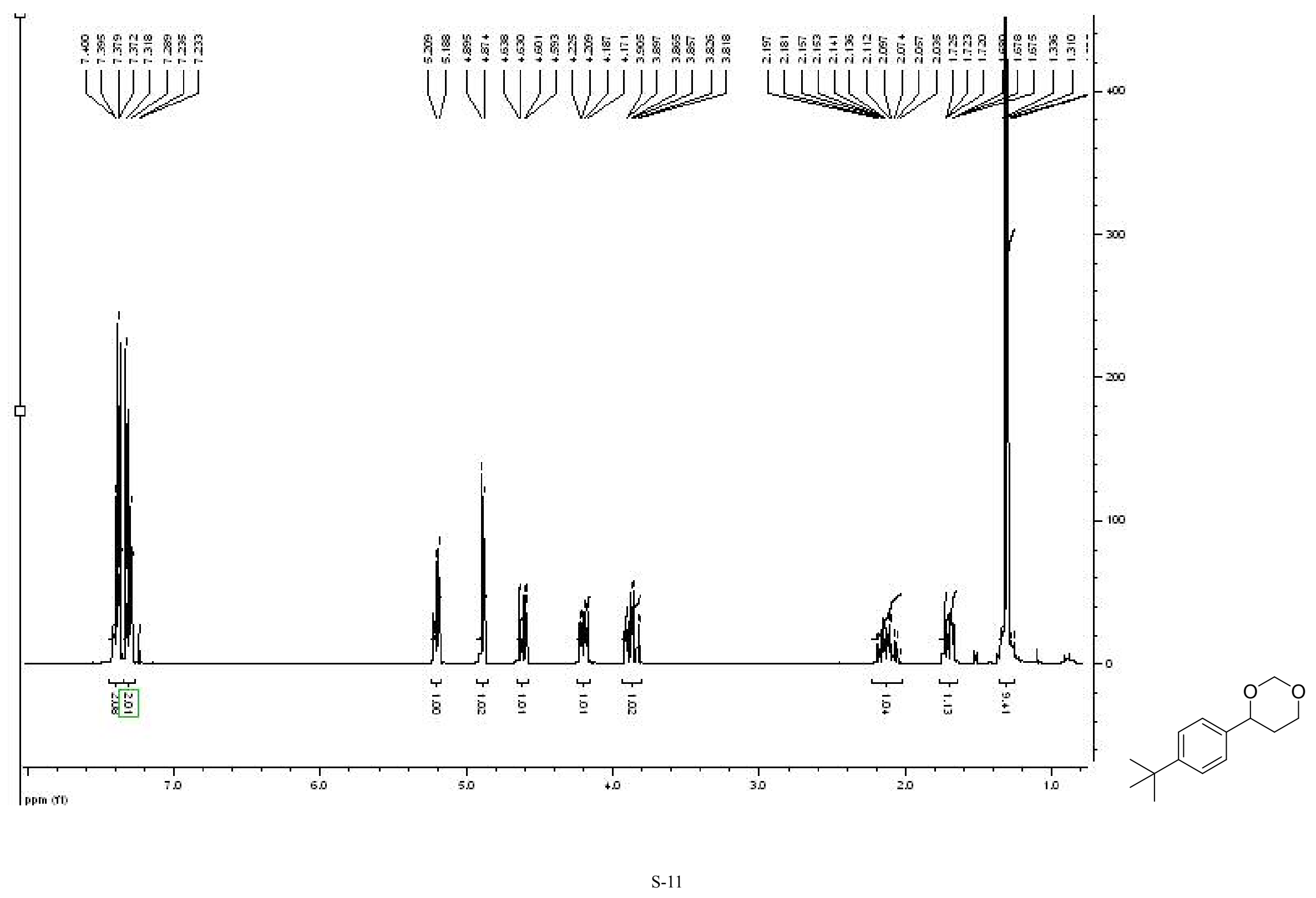



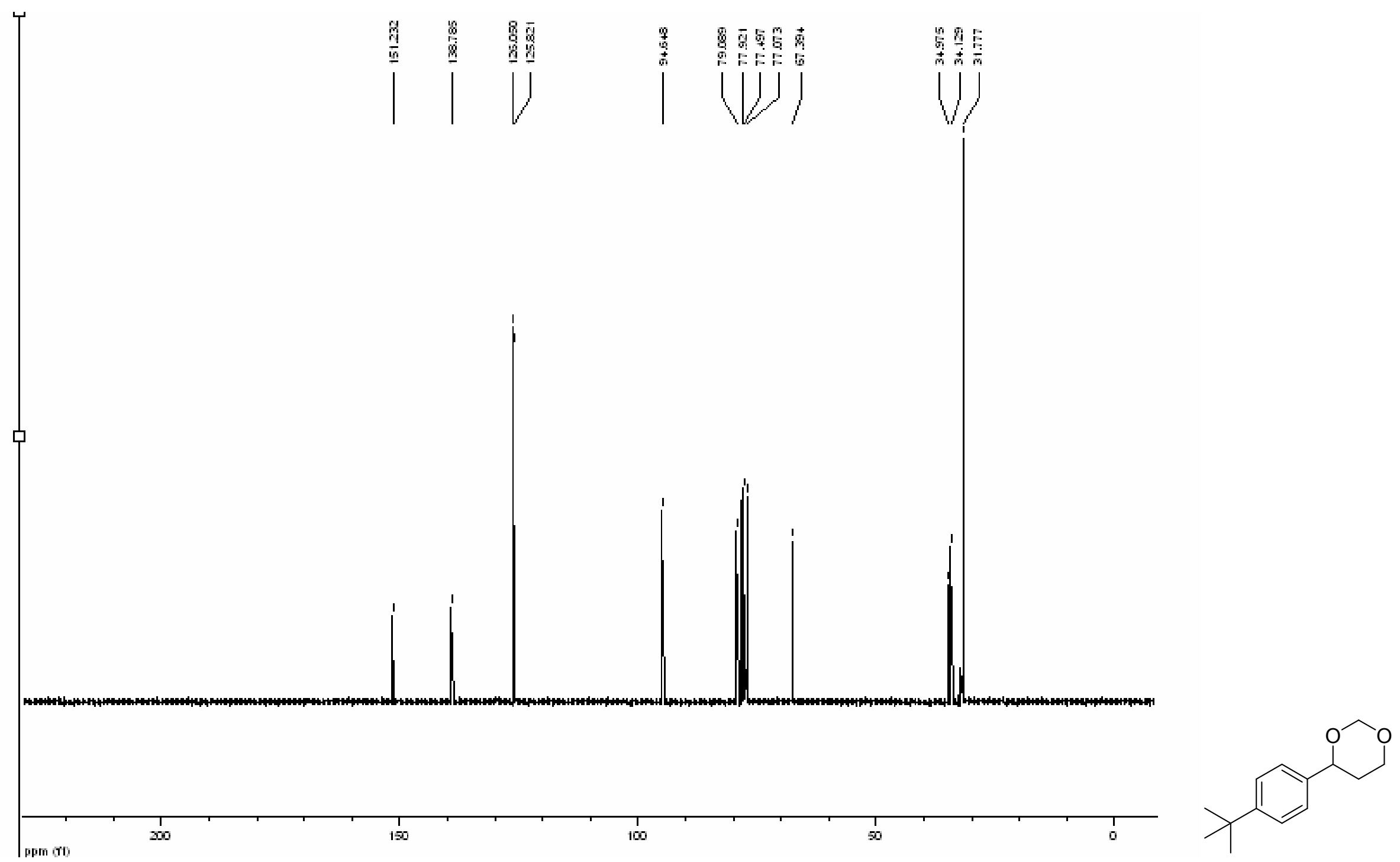


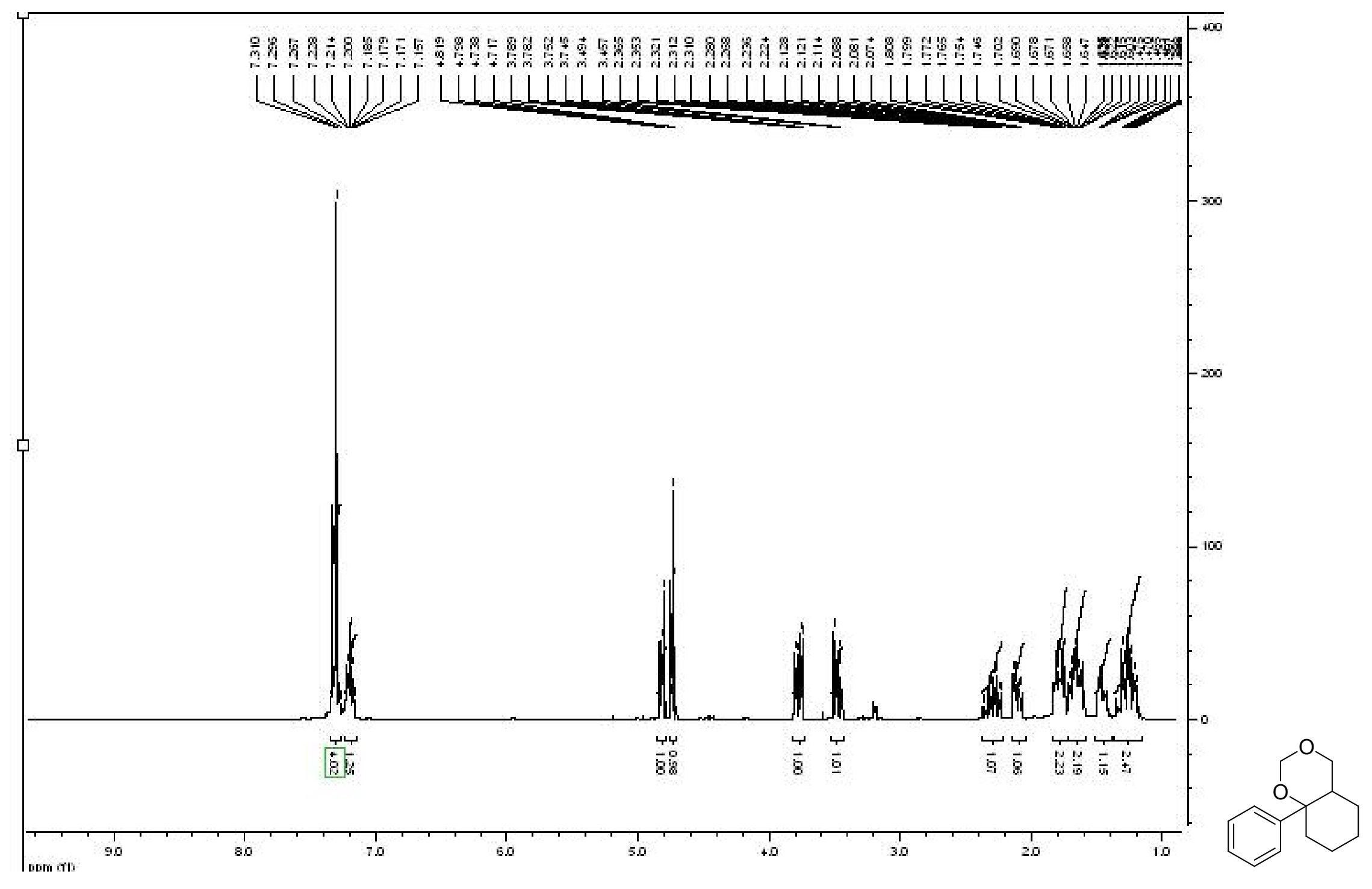



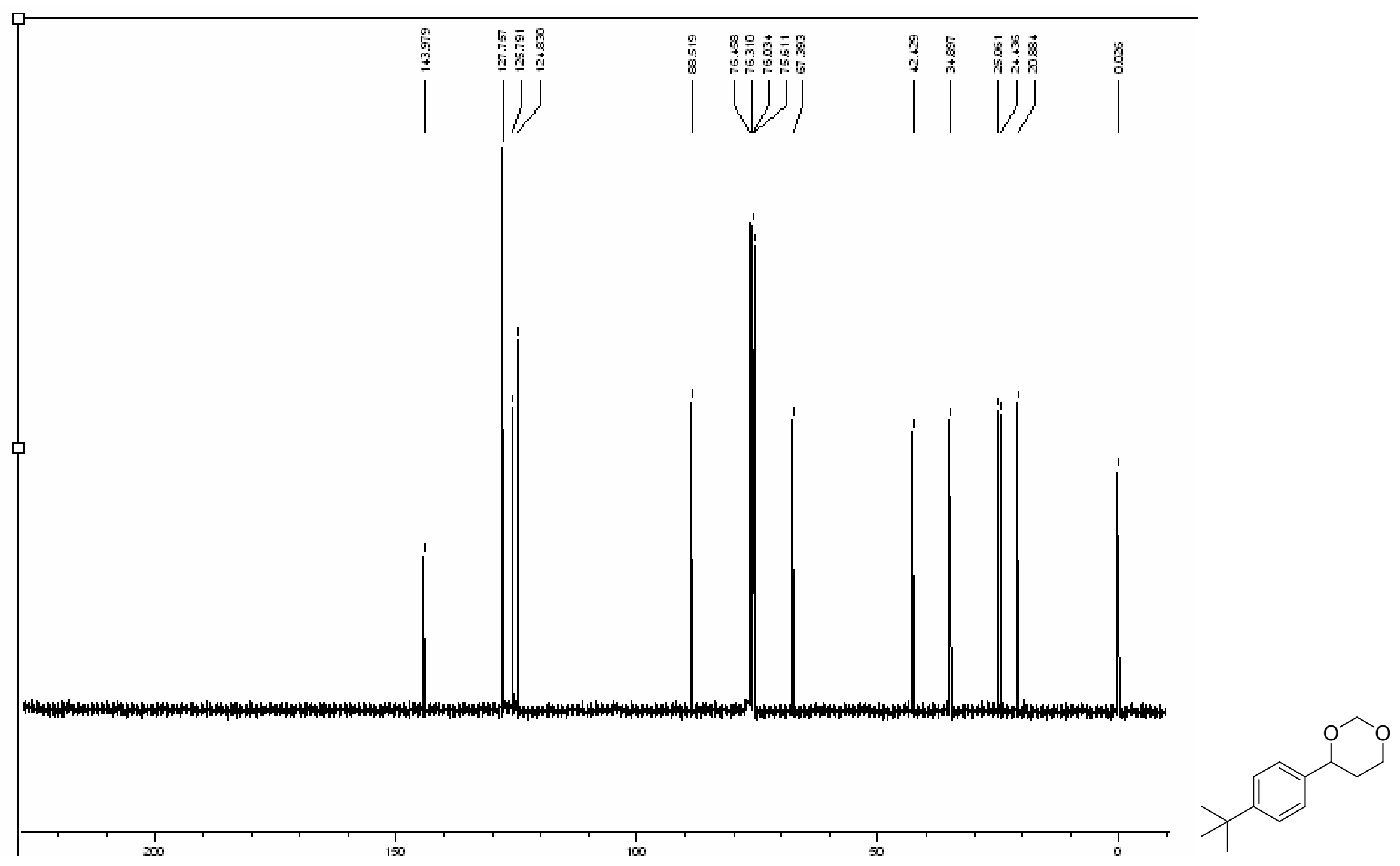


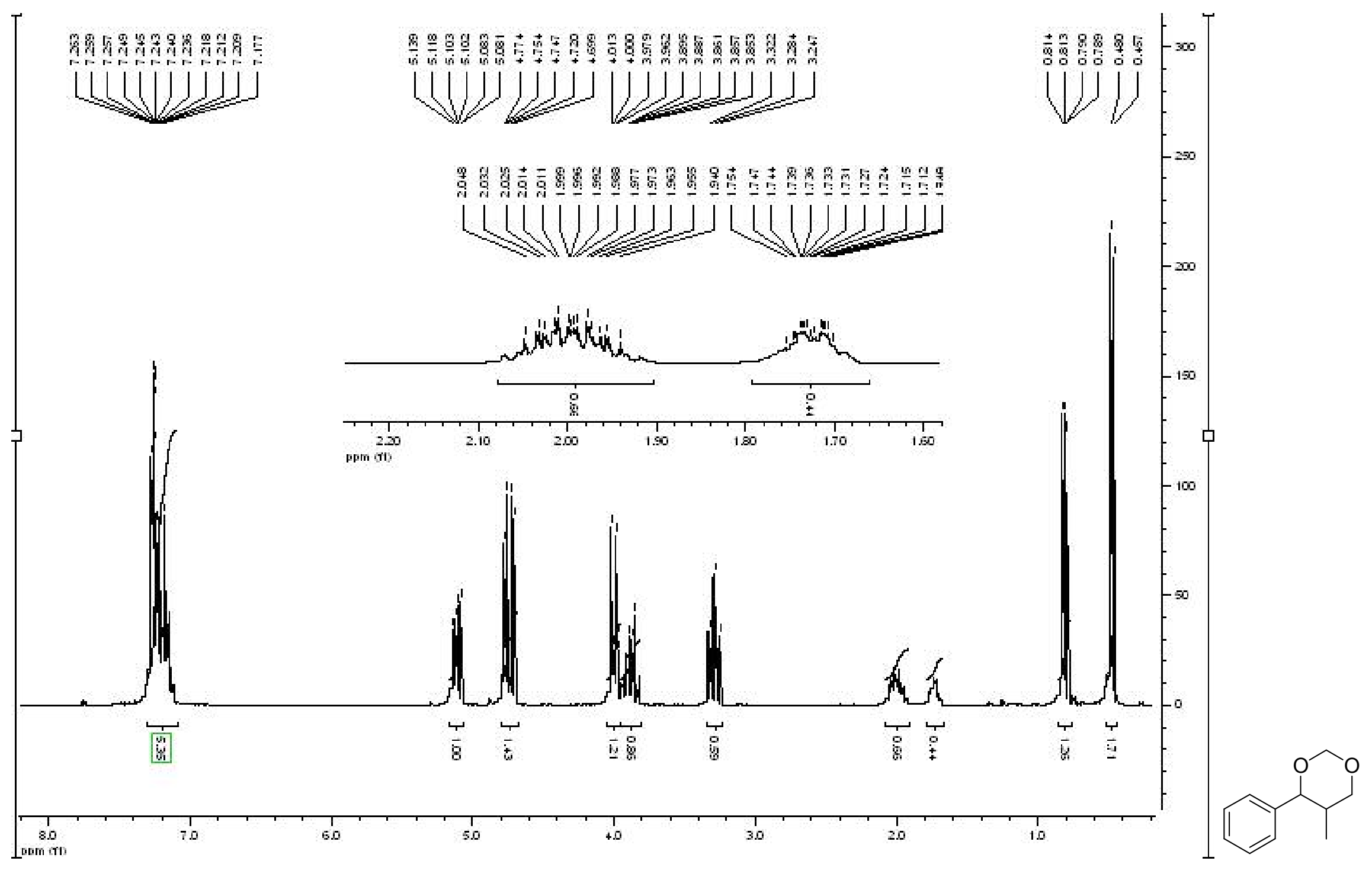




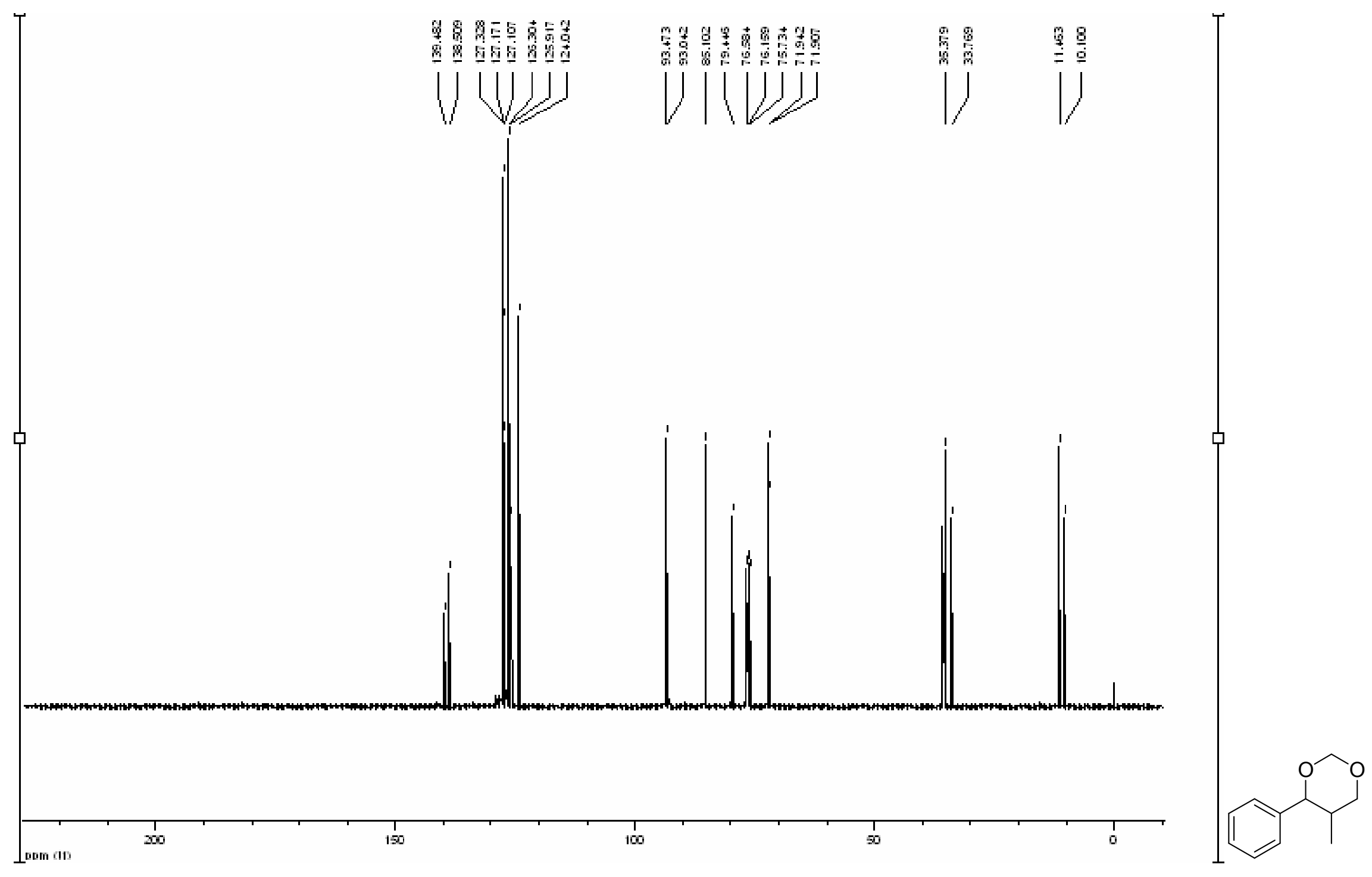




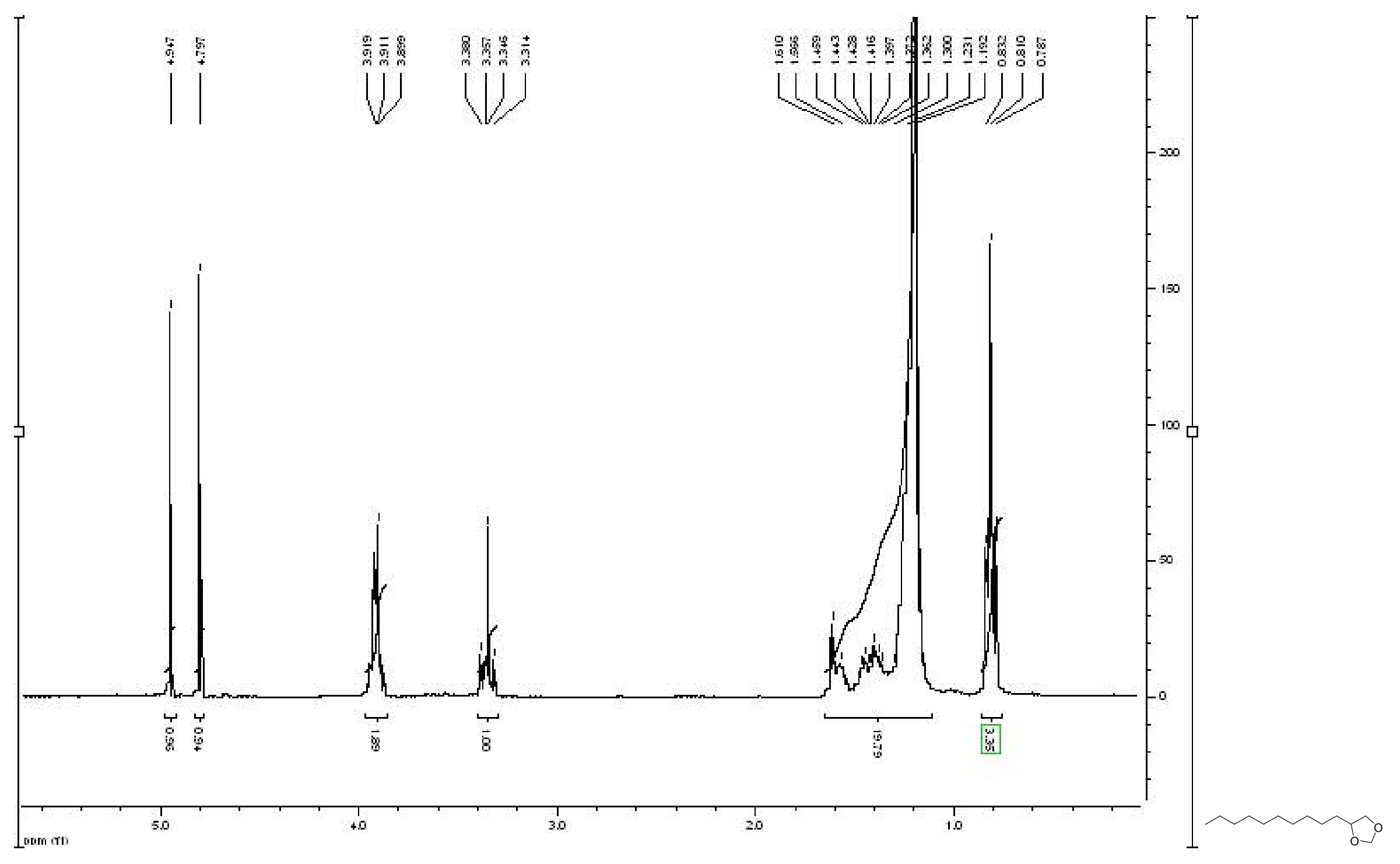




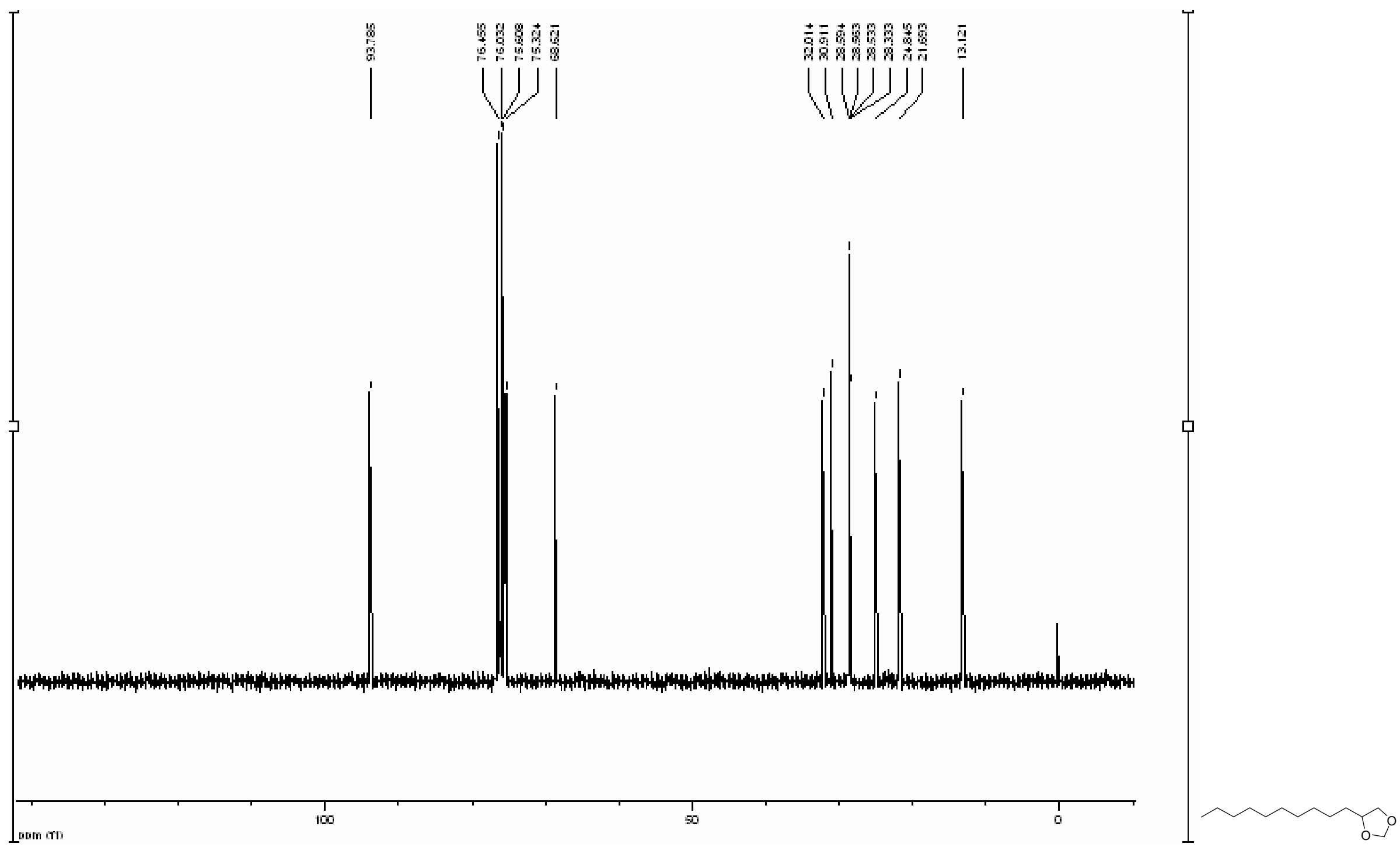

S-18 


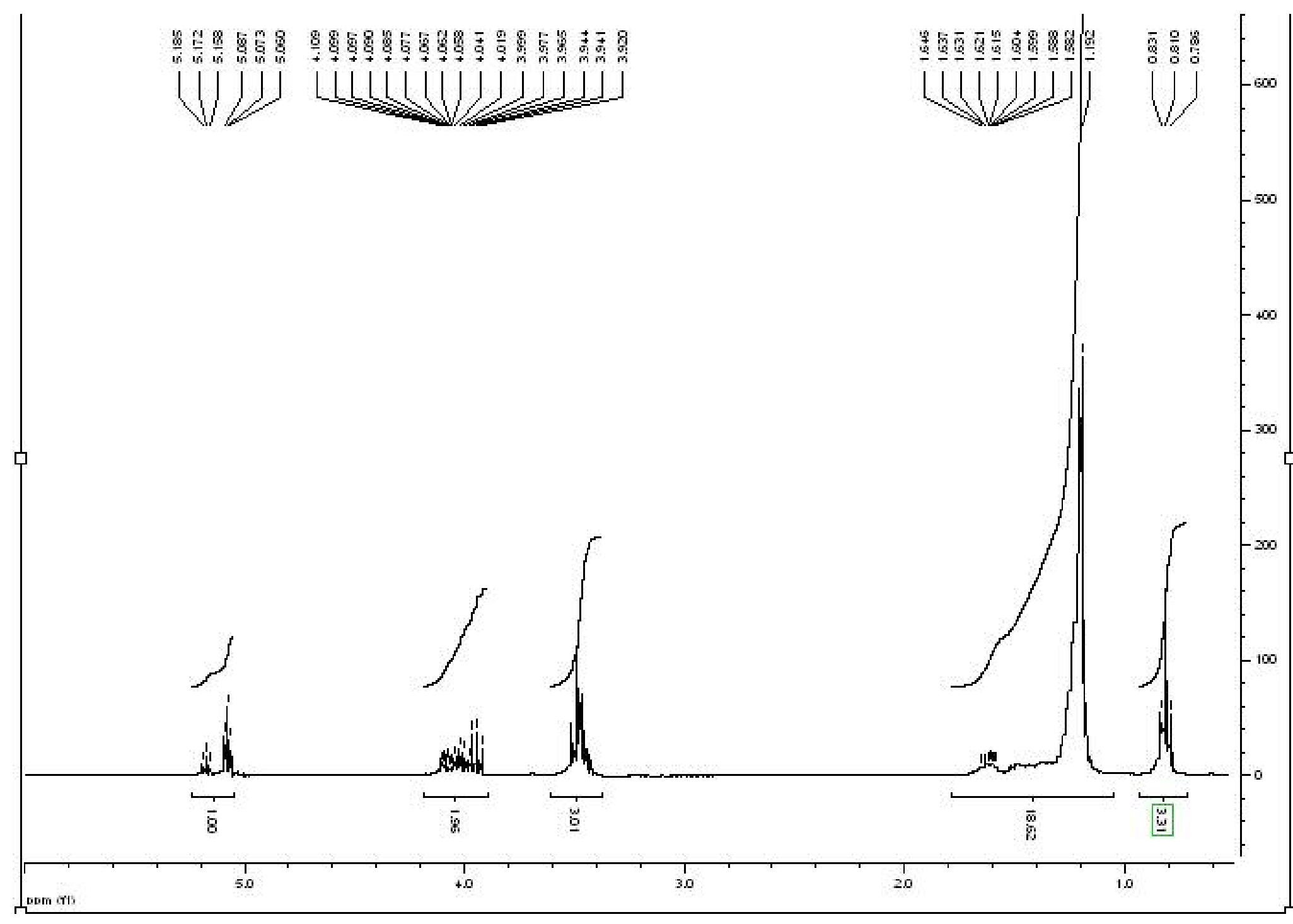

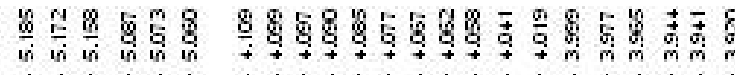

(V) $\lfloor\lfloor\lfloor\lfloor\lfloor(U) \longleftrightarrow\rfloor\rfloor\rfloor$

UUए। (U)

$+\infty$

300

200

100 


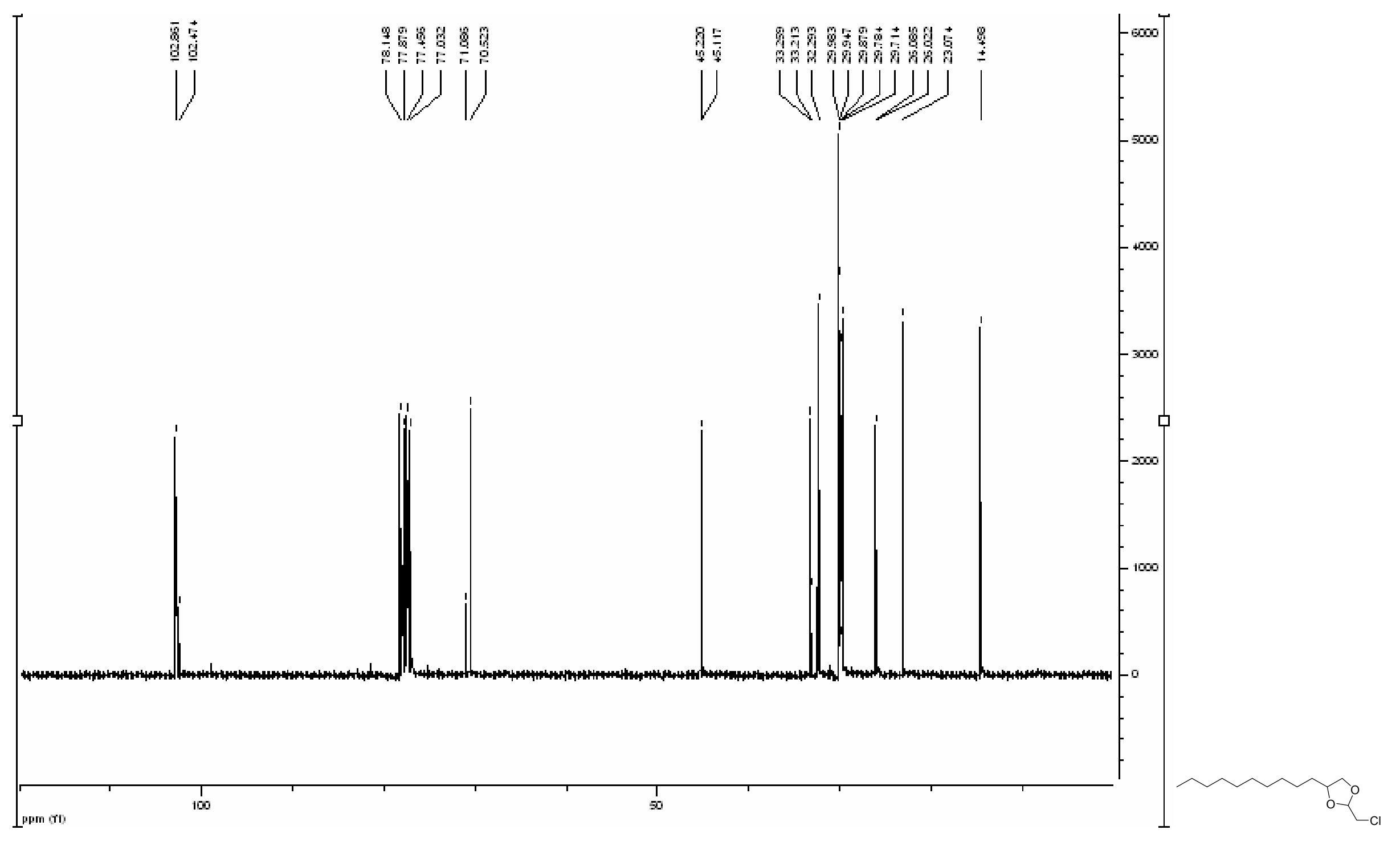




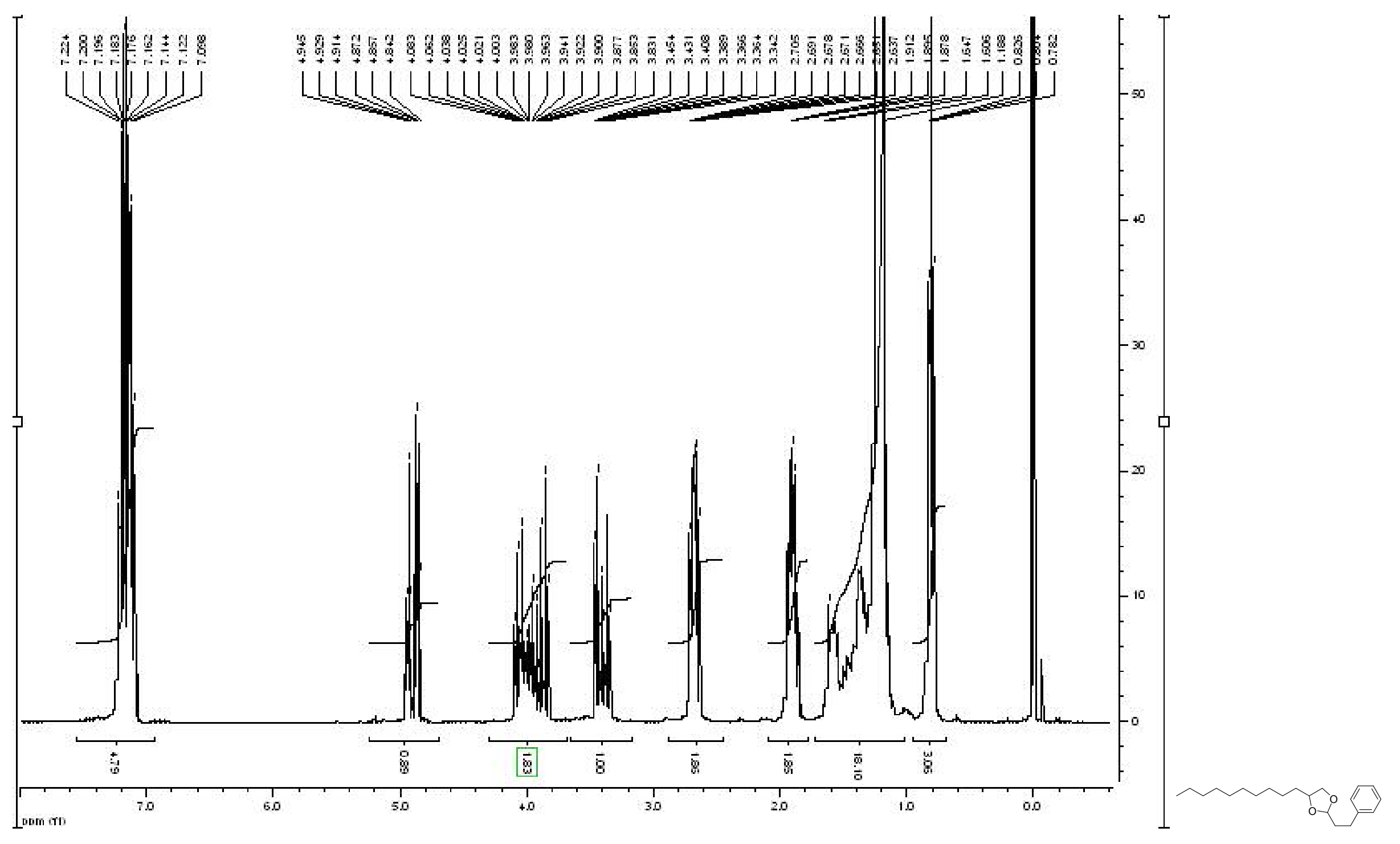




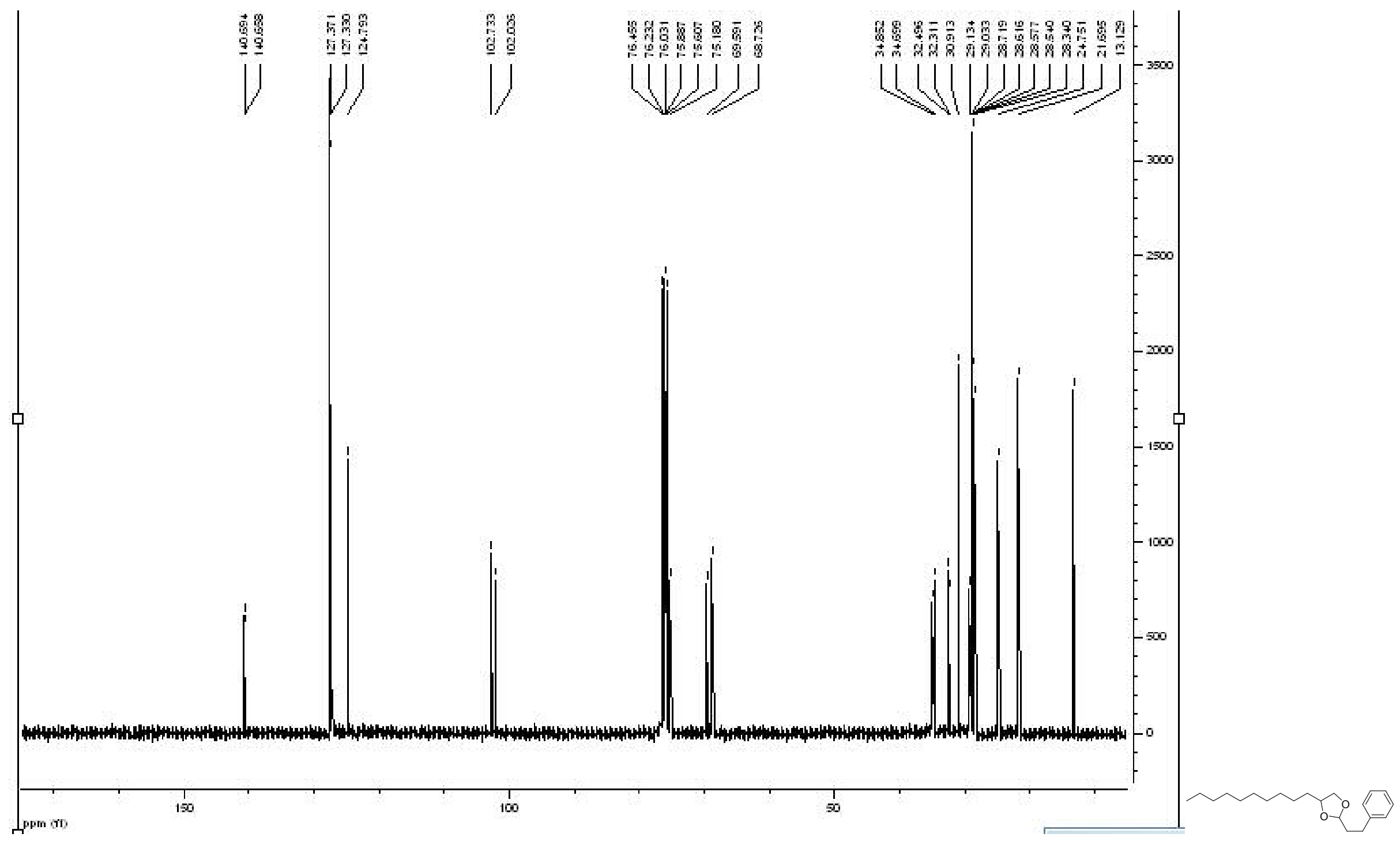




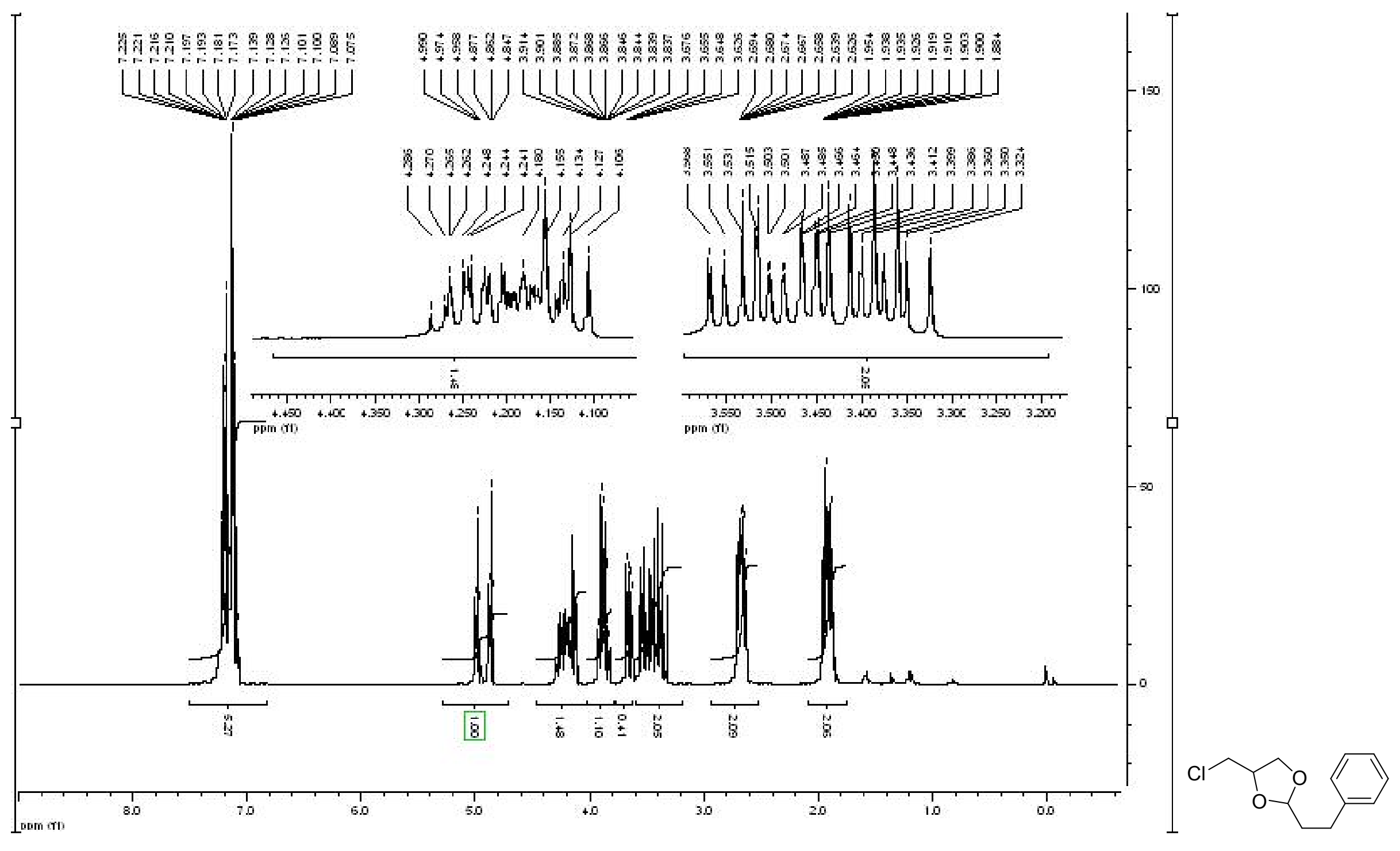




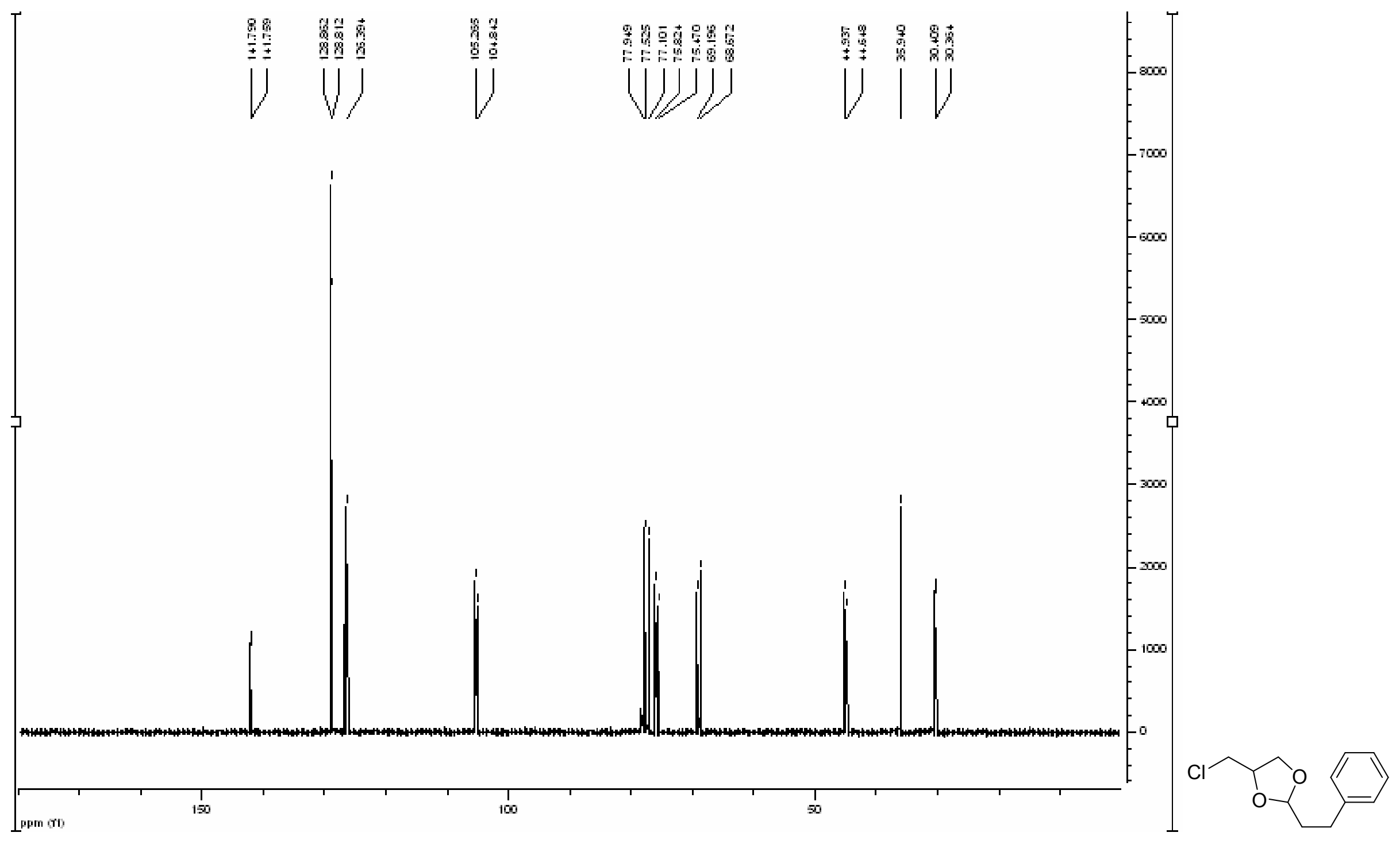




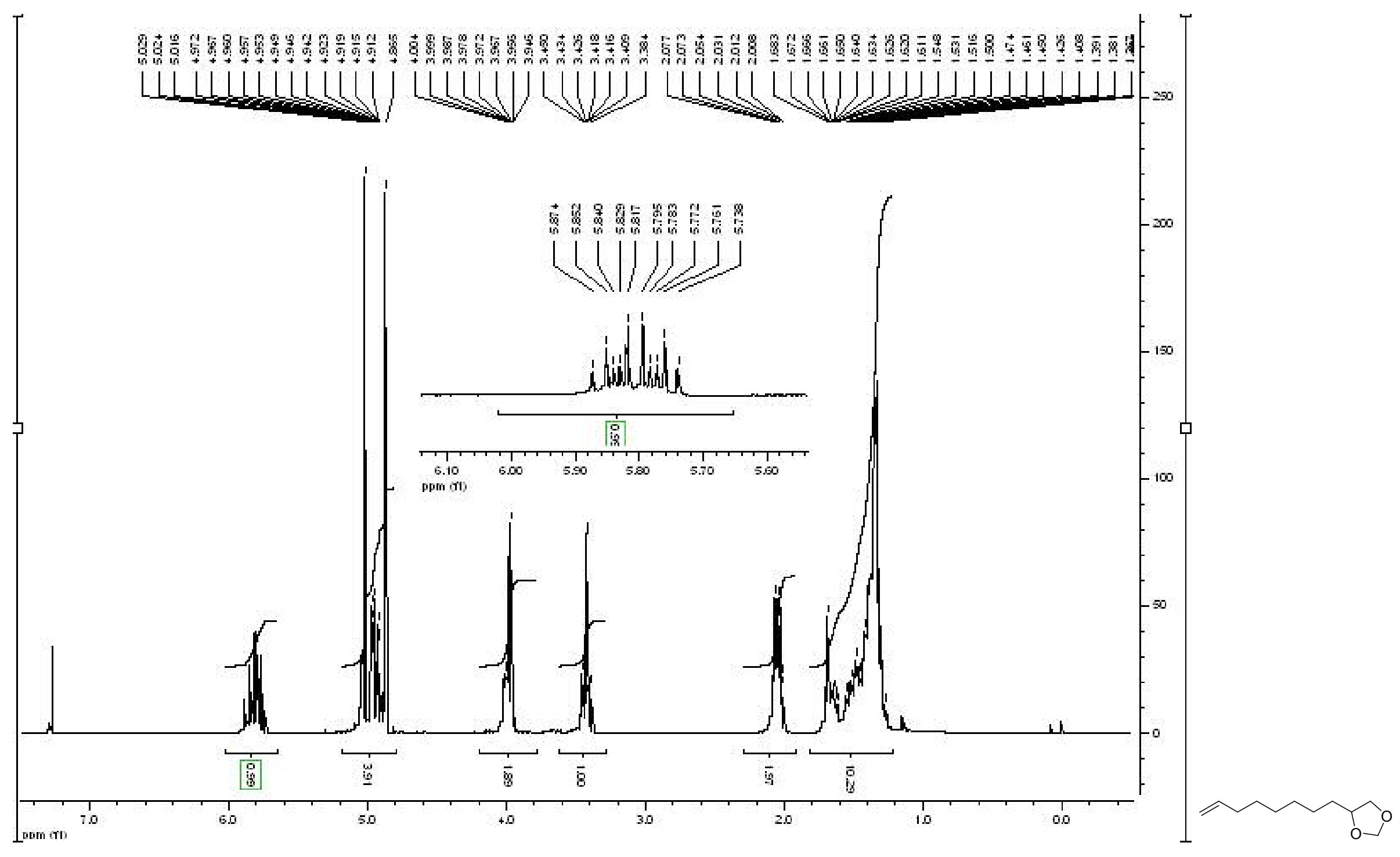




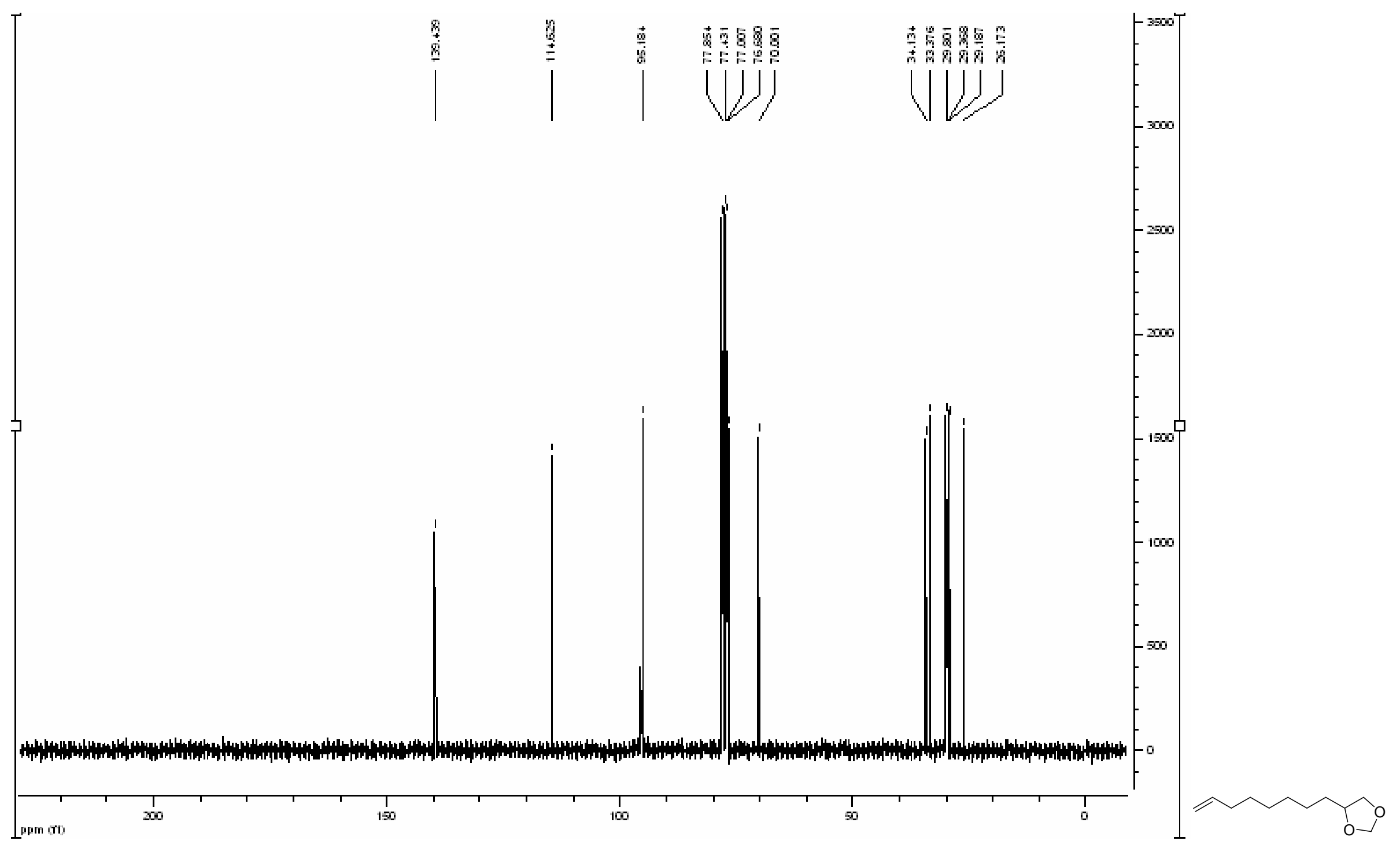




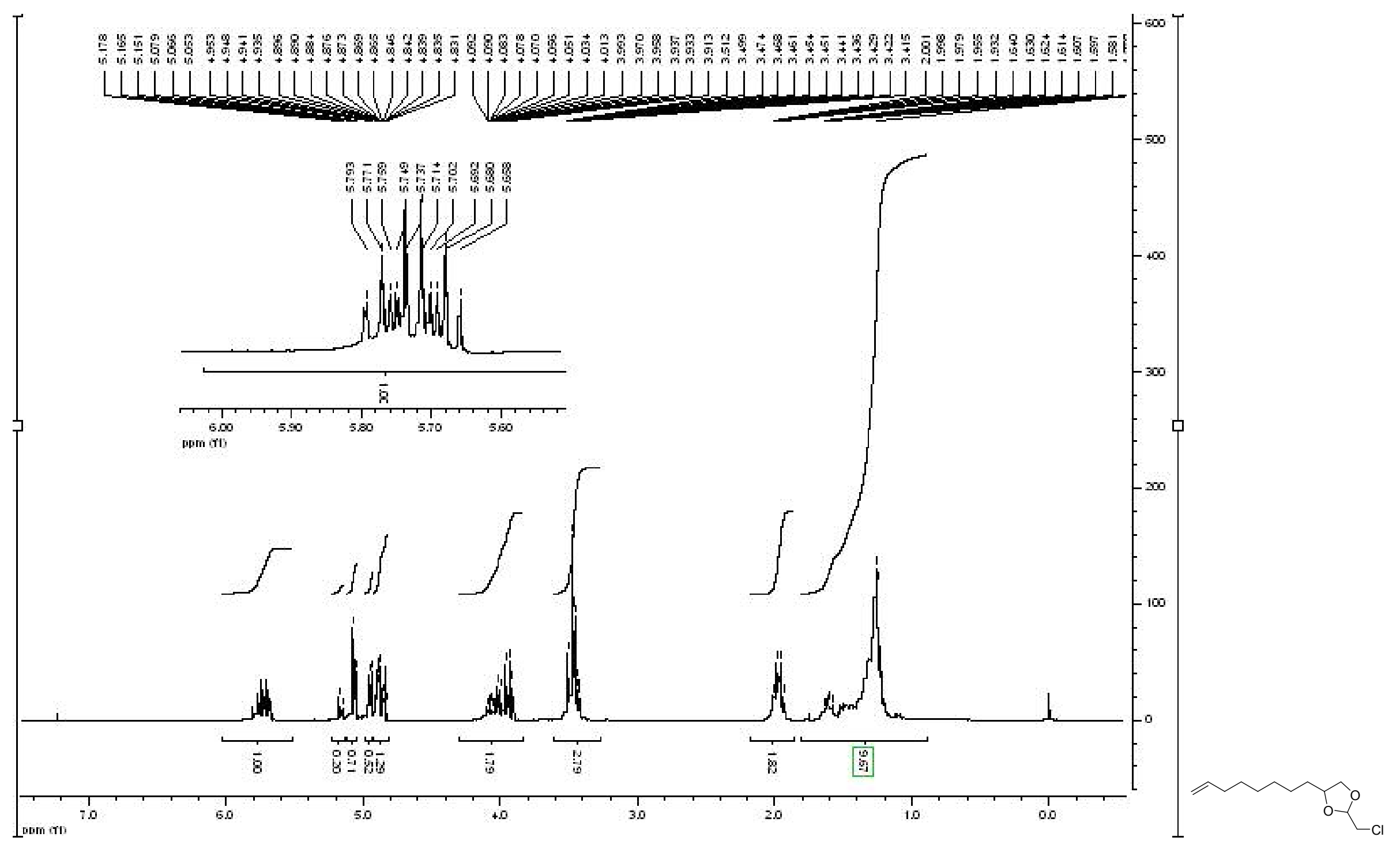




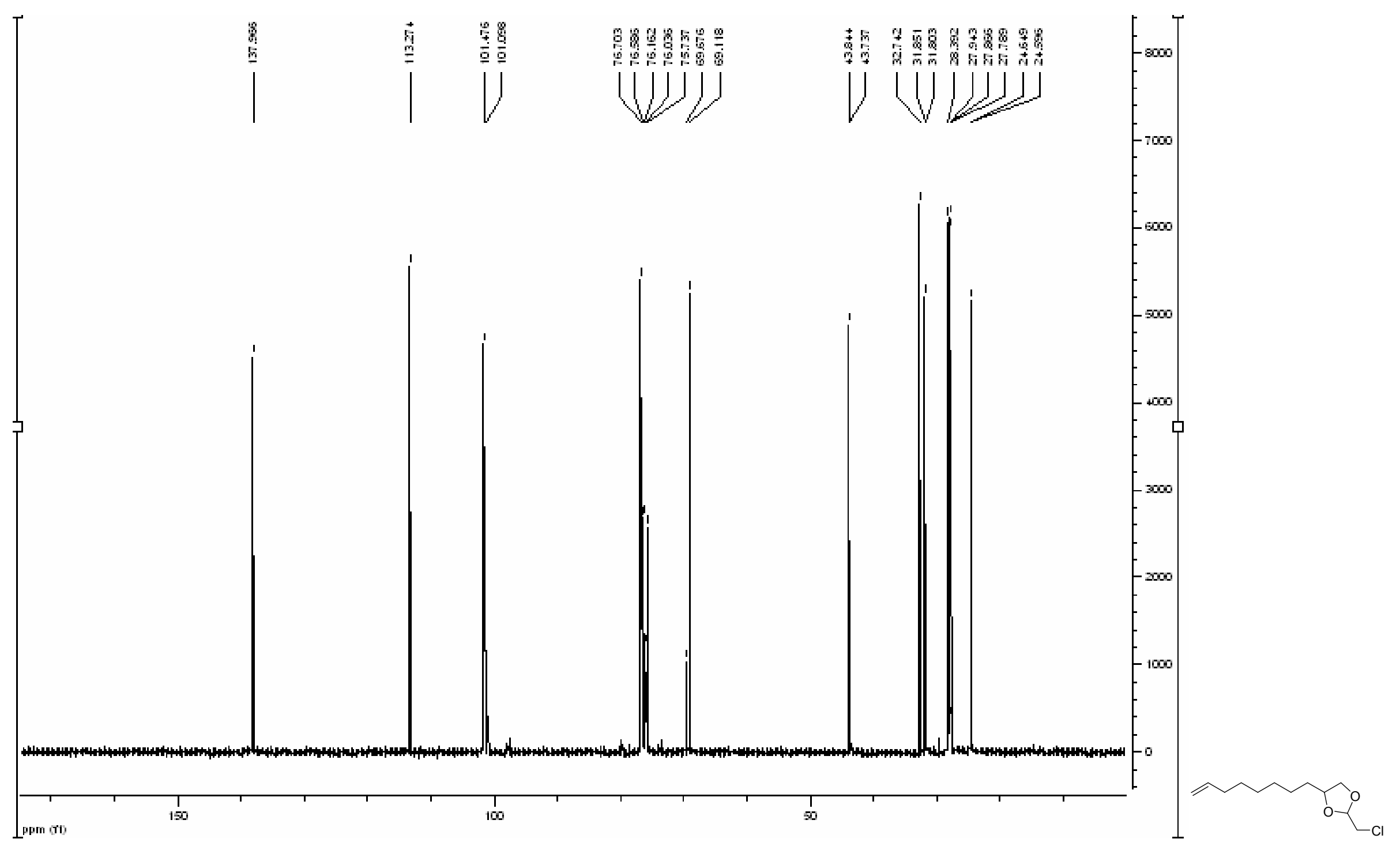




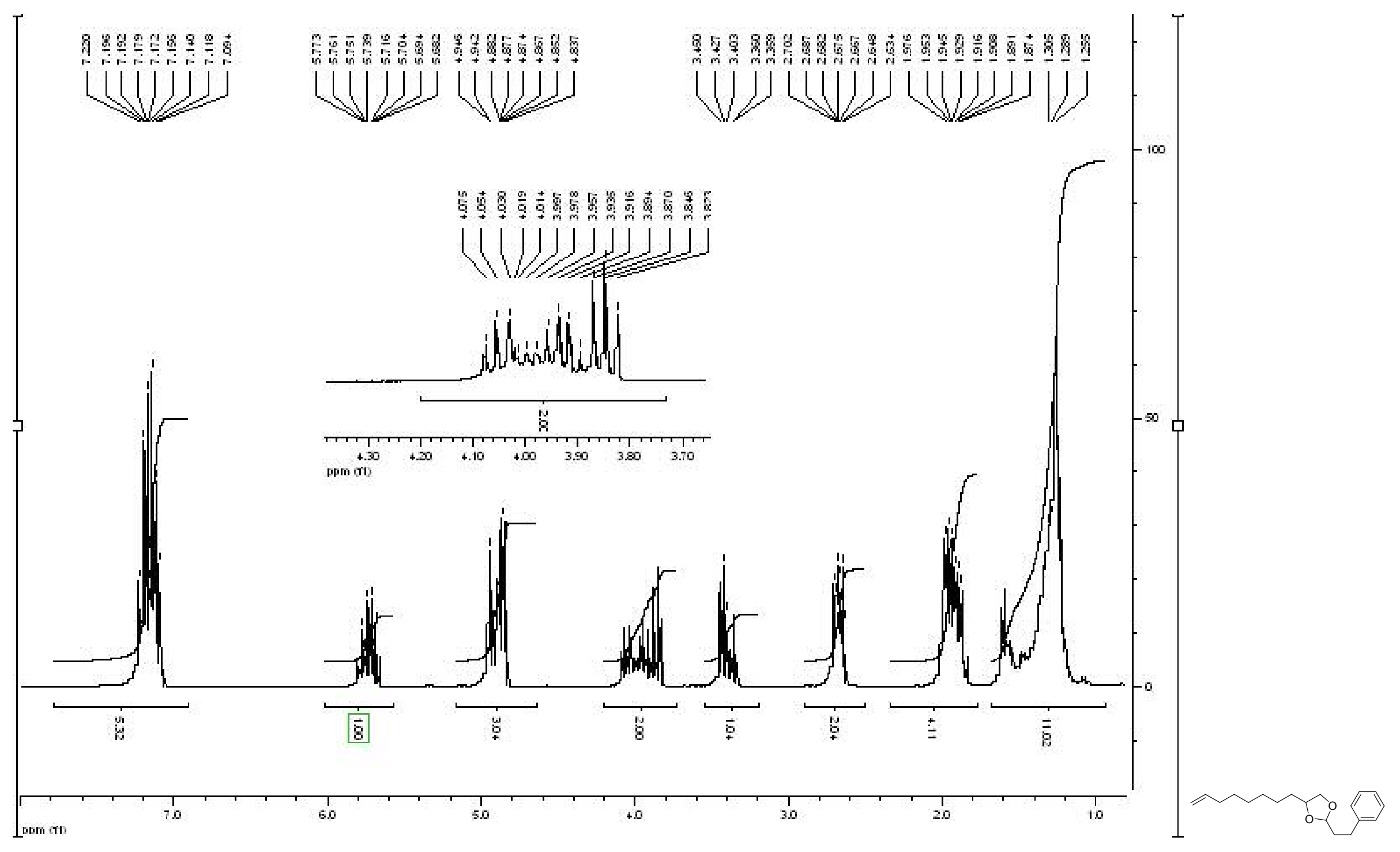




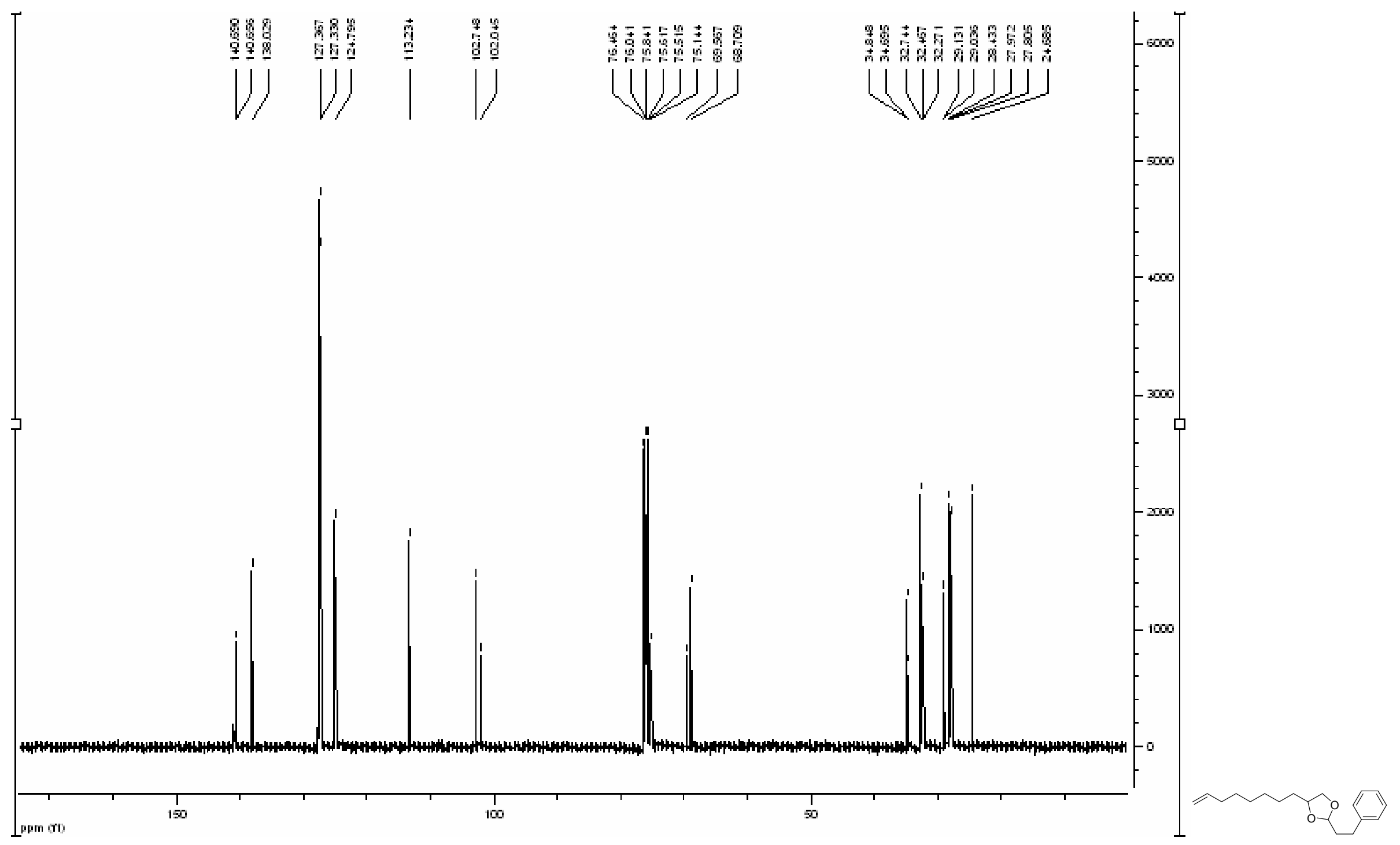




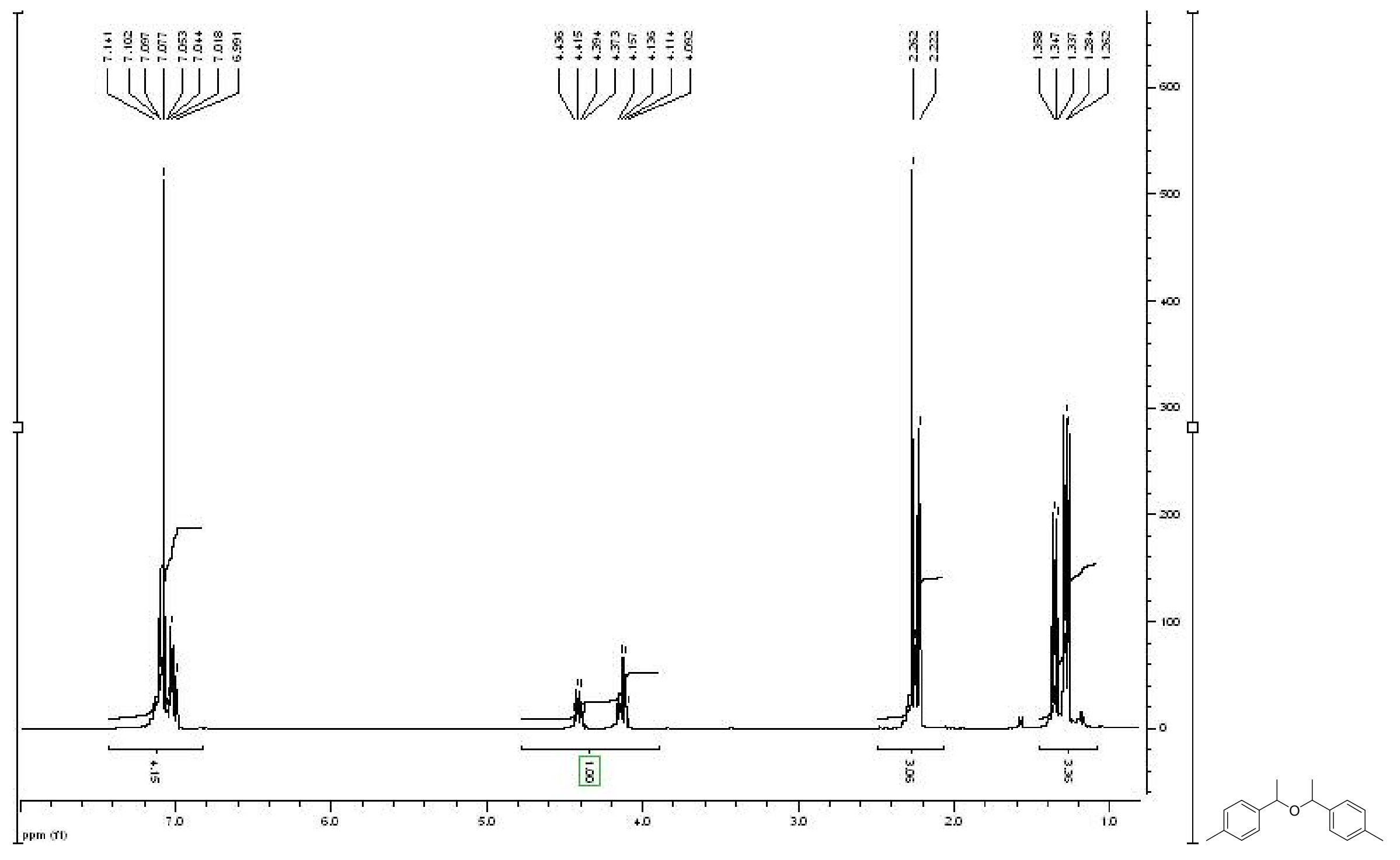




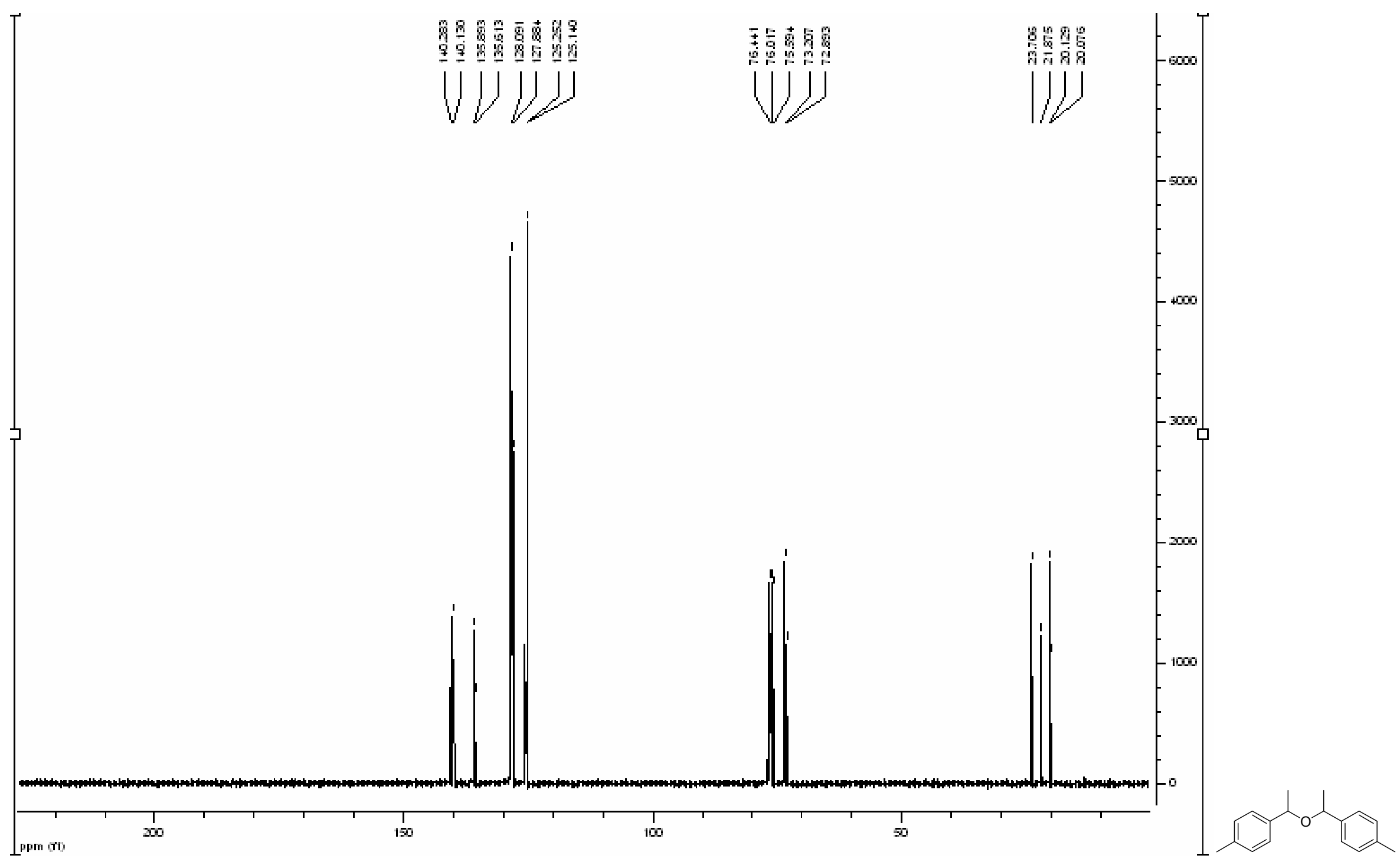




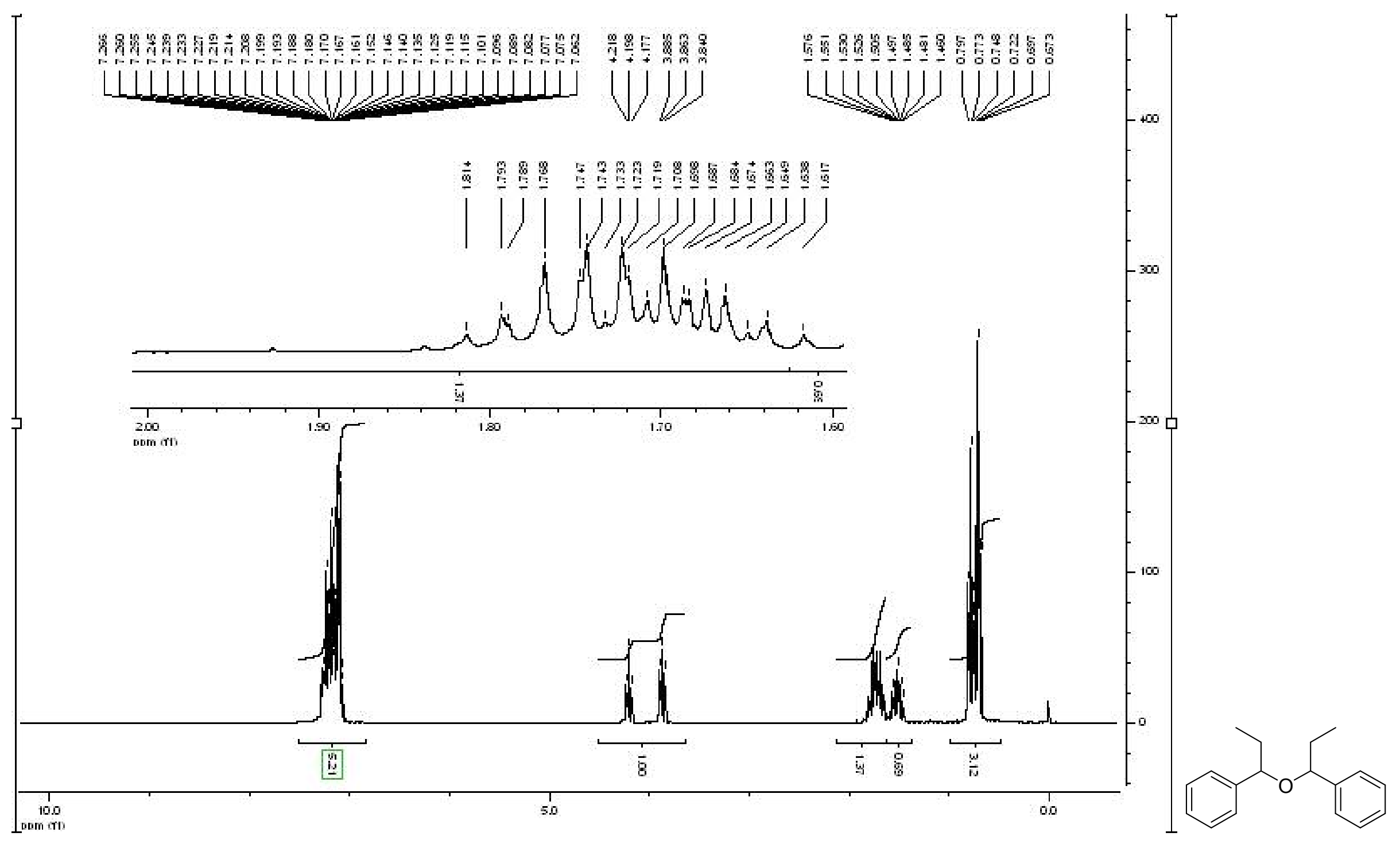




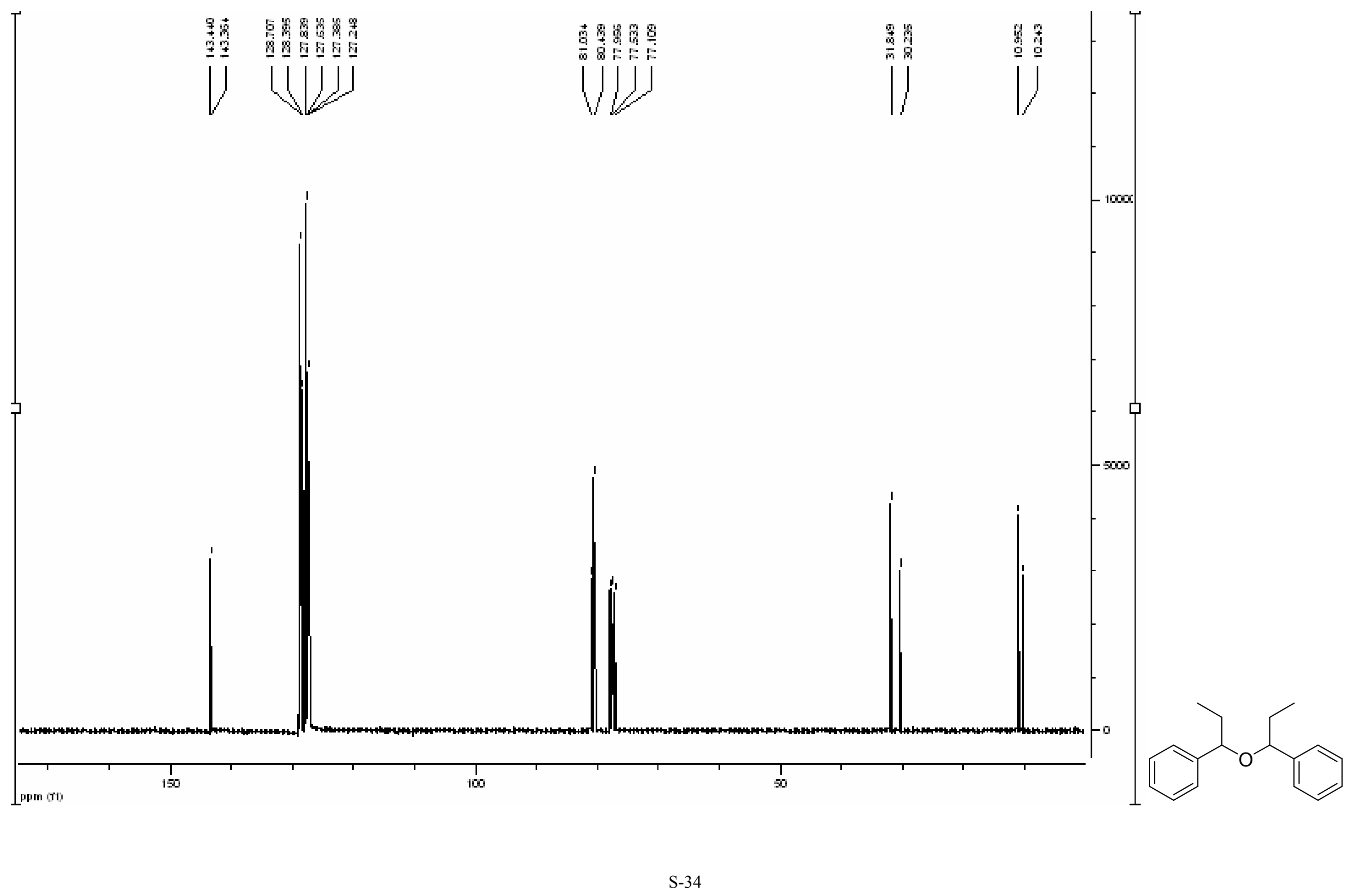




\section{References}

1 Huddleston, J. G.; Visser, A. E.; Reichert, W. M.; Willauer, H. D.; Broker, G. A.; Rogers, R. D. Green Chem. 2001, 3,156

2 Gupta, R.; Paul, S.; Gupta, R. J. Mol. Catal. A: Chem. 2007, 266, 50.

3 Gu, Y.; Ogawa, C.; Kobayashi, S.; Org. Lett. 2007, 9, 175.

4 Gu, Y.; Ogawa, C.; Kobayashi, J.; Mori, C.; Kobayashi, S. Angew. Chem, Eng. Ed, 2006, 45, 7217.

5 Chandrasekhar, S.; Reddy, B. V. S. Synlett, 1998, 851.

6 Shortridge, R. W. J. Am. Chem. Soc. 1948, 70, 873.

7 Bach, T.; Lobel, J. Synthesis, 2002, 2521.

8 Sreedhar, B.; Swapna, V.; Sridhar, C.; Saileela, D.; Sunitha, A. Synth. Commun. 2005, 35, 1177.

9 Tateiwa, J.; Hashimoto, K.; Yamauchi, T.; Uemura, S. Bull. Chem. Soc. Jpn. 1996, 69, 2361.

10 Bomhard, A.; Schaefer, M.; Markert, T.; Porrmann, V. Ger. Offen. DE 4404632, 1995.

11 Lippi, G.; Macchia, B.; Pannocchia, M. Gazzetta Chimica Italian, 1970, 100, 14.

12 Delmas, M.; Gaset, A. J. Mol. Catal. 1982, 17, 51.

13 Rosenthal, I.; Elad, D. J. Org. Chem. 1968, 33, 805.

14 Bailey, W. J.; Wu, S. R.; Ni, Z. J. Macromol. Sci. Chem. 1982, A18, 973.

15 Mattay, J.; Thuenker, W.; Scharf, H. D. Liebigs Annalen der Chemie, 1981, 1105.

16 Miller, K. J.; Abu-Omar, M. M. Eur. J. Org. Chem. 2003, 1294.

17 Mukaiyama, T.; Kuwahara, M.; Izawa, T.; Ueki, M. Chem. Lett. 1972, 287. 